\title{
Direct Observation of Laser Filamentation in High-Order Harmonic Generation
}

John Charles Painter

Brigham Young University - Provo

Follow this and additional works at: https://scholarsarchive.byu.edu/etd

Part of the Astrophysics and Astronomy Commons, and the Physics Commons

\section{BYU ScholarsArchive Citation}

Painter, John Charles, "Direct Observation of Laser Filamentation in High-Order Harmonic Generation" (2006). Theses and Dissertations. 433.

https://scholarsarchive.byu.edu/etd/433

This Thesis is brought to you for free and open access by BYU ScholarsArchive. It has been accepted for inclusion in Theses and Dissertations by an authorized administrator of BYU ScholarsArchive. For more information, please contact scholarsarchive@byu.edu, ellen_amatangelo@byu.edu. 


\title{
DIRECT OBSERVATION OF LASER FILAMENTATION IN HIGH-ORDER HARMONIC GENERATION
}

\author{
by \\ John Painter \\ A thesis submitted to the faculty of \\ Brigham Young University \\ in partial fulfillment of the requirements for the degree of
}

Master of Science

Department of Physics and Astronomy

Brigham Young University

August 2006 
Copyright (c) 2006 John Painter

All Rights Reserved 


\section{BRIGHAM YOUNG UNIVERSITY}

\section{GRADUATE COMMITTEE APPROVAL}

of a thesis submitted by

\section{John Painter}

This thesis has been read by each member of the following graduate committee and by majority vote has been found to be satisfactory.

Date

Date

Date
Justin Peatross, Chair

Michael Ware

David Neilsen 


\section{BRIGHAM YOUNG UNIVERSITY}

As chair of the candidate's graduate committee, I have read the dissertation of John Painter in its final form and have found that (1) its format, citations, and bibliographical style are consistent and acceptable and fulfill university and department style requirements; (2) its illustrative materials including figures, tables, and charts are in place; and (3) the final manuscript is satisfactory to the graduate committee and is ready for submission to the university library.

Date

Justin Peatross

Chair, Graduate Committee

Accepted for the Department

Scott D. Sommerfeldt, Chair

Department of Physics and Astronomy

Accepted for the College

Thomas W. Sederberg, Associate Dean

College of Mathematics and Physical Sciences 


\begin{abstract}
DIRECT OBSERVATION OF LASER FILAMENTATION IN HIGH-ORDER HARMONIC GENERATION
\end{abstract}

\author{
John Painter \\ Department of Physics and Astronomy \\ Master of Science
}

We investigate the spatial evolution of an intense laser pulse as it generates high-order harmonics in a long gas cell, filled with 80 torr of helium. A thin foil separates the gas-filled region of the cell from a subsequent evacuated region. The exit plane of the gas cell can be scanned along the laser axis so that the evolution of the laser throughout the focus can be observed (full scanning range of $9 \mathrm{~cm}$ ). We constructed an apparatus that images the laser radial energy profile as it exits the cell. The high harmonics, odd orders ranging from 45 to 91 , are observed at the same time that the laser spot is characterized. Re-absorption of the harmonics within the gas cell restricts the region of harmonic emission to the final centimeter (or less) of the cell.

We present the first direct evidence (to our knowledge) of laser filamentation under conditions ideal for high-order harmonic generation. The $30 \mathrm{fs}, 4 \mathrm{~mJ}$, laser pulses were observed to undergo double focusing within the gas cell, with about $4 \mathrm{~cm}$ separating the two foci. The region with best harmonic emission occurs midway between the 
two foci. The radial profile of the laser focus, 150-200 microns in diameter, evolves from a Gaussian-like profile to a more square-top profile as it propagates over several centimeters. The filamentation phenomenon as well as the brightness of the harmonics improves when an aperture is partially closed on the laser beam before reaching the focusing mirror.

A spectral sampling of the imaged laser focus revealed a $4 \mathrm{~nm}$ blue-shift associated with the generation of plasma in the gas cell. The blue-shifting occurs primarily in the center of the laser beam and less at the wider radii. The initial laser pulse had a spectrum centered at $800 \mathrm{~nm}$ with a $35 \mathrm{~nm}$ bandwidth. The energy associated with each of the observed 26 harmonic beams was found to be approximately $1 \mathrm{~nJ}$, yielding a conversion efficiency of approximately $2 \times 10^{-7}$. 


\section{ACKNOWLEDGMENTS}

I would like to express appreciation to Justin Peatross, my advisor, for his support, insight and friendship. He has expected great things from me and given me many opportunities to fulfill them. I am also grateful to David Neilsen and Michael Ware, members of my committee, for their contributions and suggestions.

Mark Adams, Gavin Giraud, Nathan Powers, and Matt Turner all made helpful contributions to this research. I am especially grateful to Niki Brimhall for her long hours in the lab. Without her assistance, this thesis would not have happened.

I am thankful for my parents who have always believed I was capable of accomplishing great tasks and encouraged me to try. My father's bits of wisdom continually lifted up my spirits.

Finally, I want to thank my wife, Deborah, and my daughter, Eleni, for their love and support during what has been a difficult, time-involved and daunting task. They motivated and encouraged me when things were not going as well as I had hoped they would. 



\section{Contents}

Table of Contents $\quad$ ix

List of Figures $\quad$ xi

1 Introduction $\quad 1$

1.1 Extreme Utlraviolet Light . . . . . . . . . . . . . . . . . . . . 1

1.2 High-Order Harmonic Generation . . . . . . . . . . . . . . . 3

1.2.1 Conversion Efficiency . . . . . . . . . . . . . . 5

1.3 Phase-Matching . . . . . . . . . . . . . . . . 6

1.4 Phase Matching Techniques . . . . . . . . . . . . . . . 8

1.5 Filamentation . . . . . . . . . . . . . . . . . . . . . . . . . . . . . .

1.6 Previous High-Harmonic Work at BYU . . . . . . . . . . . . . . . 11

1.7 Outline of Experiments . . . . . . . . . . . . . . . . . . . . . . . . . . . . . . . . . . . . . 13

1.8 Thesis Overview . . . . . . . . . . . . . . . . . . 14

2 Experimental Setup and Procedures 17

2.1 Laser System . . . . . . . . . . . . . . . . . . . . . . 17

2.2 Experimental Setup . . . . . . . . . . . . . . . . . . . . 20

2.3 Imaging Apparatus . . . . . . . . . . . . . . . . . . . . . . . . . . . . . . . . . . . 22

2.4 Alignment of the Imaging Apparatus . . . . . . . . . . . . . . . . . . 25

2.5 Optimizing Parameters for High Harmonic Production . . . . . . . . 27

3 Evolution of Laser Spatial Profile 29

3.1 Harmonic Generation at Different Gas-Cell Positions . . . . . . . . . 29

3.2 Apertured Laser Beam . . . . . . . . . . . . . . . . . . . . 30

3.3 Laser Propagation in Gas Versus in Vacuum . . . . . . . . . . . . . . 34

3.4 Beam-Width Measurements . . . . . . . . . . . . . . . 39

4 Auxiliary Measurements $\quad 47$

4.1 Pulse Duration . . . . . . . . . . . . . . . . . . . . . . 47

4.2 Laser Focus Spectral Content . . . . . . . . . . . . . . . . . . 48

4.3 Harmonic Energy Measurement . . . . . . . . . . . . . . . 50

5 Conclusions $\quad 57$ 
Bibliography 


\section{List of Figures}

1.1 Depiction of how harmonics are generated in gas . . . . . . . . . . . 4

1.2 Shorter wavelengths diffract more slowly as they travel through the focus 7

1.3 Counter-propagating pulses $\mathrm{Ne}$ and $\mathrm{He}$. . . . . . . . . . . . . . 12

2.1 Near field image of laser beam showing non-uniformity of the structure 18

2.2 Laser-beam profile at focus . . . . . . . . . . . . . . . . 18

2.3 Auto-correlation image of the laser pulse . . . . . . . . . . . . . . . . 19

2.4 Harmonic generation setup . . . . . . . . . . . . . . . . . . . . 20

2.5 Harmonics generated in 80 torr helium . . . . . . . . . . . . . . 21

2.6 Laser beam image taken $36 \mathrm{~cm}$ before focus . . . . . . . . . . . . 23

2.7 Laser beam image taken a focus . . . . . . . . . . . . . . . . . . 23

2.8 Imaging Apparatus . . . . . . . . . . . . . . . . . . . . . . 24

2.9 Near field focus compared to imaged focus in vacuum . . . . . . . . 25

2.10 Foil pinhole used in alignment . . . . . . . . . . . . . . . 26

3.1 Image of harmonics with beam profile and lineout . . . . . . . . . . . 31

3.2 Image of harmonics with beam profile and lineout . . . . . . . . . . . 32

3.3 The intensity of harmonics with a slightly apertured beam was increased by almost a factor of 3 over the open aperture case. . . . . . . 33

3.4 Image of harmonics with beam profile and lineout . . . . . . . . . . 35

3.5 Image of harmonics with beam profile and lineout . . . . . . . . . . 36

3.6 Image of harmonics with beam profile and lineout . . . . . . . . . . . 37

3.7 Image of harmonics with beam profile and lineout . . . . . . . . . . 38

3.8 Beam profile for helium and vacuum at various foil positions . . . . . 40

3.9 Beam profile for helium and vacuum at various foil positions . . . . . 41

3.10 Beam profile for helium and vacuum at various foil positions . . . . . 42

3.11 Beam profile for helium and vacuum at various foil positions . . . . . 43

3.12 A graph of FWHM for both helium and vacuum for various foil positions 44

4.1 Comparison of the temporal pulse duration optimized in air versus best harmonic . . . . . . . . . . . . . . . . . . 48

4.2 Spectral content of imaged laser focus . . . . . . . . . . . . . . . 49

4.3 Photodiode assembly . . . . . . . . . . . . . . . . . . 51

4.4 Harmonic induced voltage . . . . . . . . . . . . . . . . 52 
4.5 Noise induced voltage . . . . . . . . . . . . . . . . . . . 52

4.6 Zr filter transmission curve . . . . . . . . . . . . . . . . . . . 53

4.7 Quantum efficiency of photodiode . . . . . . . . . . . 54 


\section{Chapter 1}

\section{Introduction}

\subsection{Extreme Utlraviolet Light}

The computer industry has generated strong interest in the Extreme Ultraviolet (EUV) wavelength range, since elemental circuit sizes can be made no smaller than the wavelength used in the lithograph process. Currently, the shortest wavelength used in commercial lithography is $196 \mathrm{~nm}$. There is a push to go to $13.5 \mathrm{~nm}$ wavelength, which should allow circuit elements on computer chips that are ten times smaller than currently feasible [1]. However, working in the ultraviolet spectrum poses challenges. Because EUV light is absorbed by all materials, EUV lithography must rely on reflective imaging optics instead of transmissive optics. Also, the entire light path must be in vacuum since EUV light is absorbed by air.

Synchrotrons are a valuable source of EUV light, with photon energies from a few to thousands of electron volts. However, synchrotrons are very expensive with price tags nearing a hundred million dollars and diameters the size of a football field [2]. Inside a synchrotron, electrons are accelerated around a large circular path. In order to achieve the desired wavelengths and brightness, the electron beam must be 
maintained at energies over a $\mathrm{GeV}$. The light produced in a synchrotron is a hundred million times brighter than that from the most powerful x-ray tube and exhibits tunability, with good coherence and polarization.

Another source of EUV light is emission from laser produced plasmas. Plasmabased EUV sources rely on line radiation from highly ionized atoms [3]. Xenon has a well known emission line at $13.5 \mathrm{~nm}$, which is the targeted wavelength for EUV lithography. However, the conversion efficiency for xenon is relatively low: less than $1 \%$ [4]. There is still a lot of work to be done to achieve higher conversion efficiencies with lower input powers.

A challenge in producing optics such as mirror coatings for use in the EUV range is incomplete information on the index of refraction for coating materials. Two professors in our department, David Allred and Steve Turley, are currently doing research on the design and fabrication of multilayer mirrors for the EUV. Their research group produced mirror coatings that were used on the IMAGE satellite, launched March 25, 2000 [5]. It was the first instrument to take a series of pictures of the Earths magnetosphere at a photon energy of $41 \mathrm{eV}(30 \mathrm{~nm})$ as part of a comprehensive multi-spectral imaging of the near space around the Earth. The mirror coating that they designed for this satellite needed high reflectance (greater than 20\%) at $30.4 \mathrm{~nm}$, 14-degrees from normal, and low reflectance (less than $2 \%$ ) at $58.4 \mathrm{~nm}$. Their work was hampered by the fact that the index of refraction for one or more of the materials differed from published values. This necessitated many visits to the Berkeley synchrotron source, located over 500 miles away, in order to make reflectivity measurements. A polarimeter based on EUV light co-located with the sample-preparation site would allow a more rapid turn-around production and characterization of EUV optics. 


\subsection{High-Order Harmonic Generation}

Laser high-order harmonic generation (HHG) is a useful alternative source of EUV light. Although high-order harmonic pulses have far less energy than the sources previously mentioned, they are produced as coherent, highly directional, and polarized beams that can be a useful source to aid in the development of EUV optical components.

In 1987, McPherson et al. observed harmonics of their 248-nm laser with orders as high as the seventeenth generated in neon and the thirteenth generated in helium [6]. This was the first time that harmonic orders well above the third and fifth had been generated with a laser, which marked the birth of high-order harmonic research. Their laser had a pulse energy of $20 \mathrm{~mJ}$ and pulse duration of $350 \mathrm{fs}$, which achieved $10^{15}-10^{16} \mathrm{~W} / \mathrm{cm}^{2}$ focused intensity in their focal geometry. A gas jet was used to spray the gas into the laser focus inside an otherwise evacuated chamber. This technique is still commonly used today.

Since the initial work of McPherson et al., many groups have studied HHG in an effort to achieve the highest possible conversion efficiency and the shortest possible wavelength. Many experiments have been performed using a variety of laser wavelengths, pulse durations, focused intensities, and different gas species to generate high harmonics. In a landmark study, Anne L'Hullier et al. did a broad survey of HHG using a 1 ps Nd:Glass laser with wavelength $1.054 \mu \mathrm{m}$ [7]. They observed up to the $29^{\text {th }}$ harmonic in xeon, the $57^{\text {th }}$ in argon, and and up to the $135^{\text {th }}$ in helium. They also investigated the influence of gas density and focal geometry on the conversion efficiency, which reached up to $10^{-6}$ in the case of xenon.

The laser intensity needed for HHG in helium is near $10^{15} \mathrm{~W} / \mathrm{cm}^{2}$. In the presence of the strong oscillating electric field of the laser, electrons are torn from the 


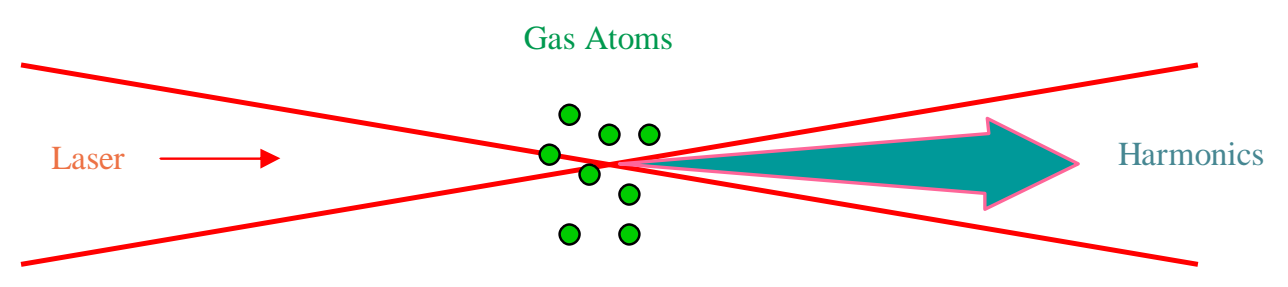

Figure 1.1 Harmonics generated in a laser focus.

helium atoms and accelerated. As the oscillating laser field reverses direction, some of the electrons return, collide, and recombine with the parent ion, which releases energetic photons with frequencies that are odd multiples of the laser frequency. A wide plateau of harmonic orders are simultaneously generated up to a maximum order associated with a cutoff frequency. The wavelength of a harmonic is $\lambda / q$ where $\lambda$ is the wavelength of the laser and $q$ is the specific harmonic order (odd integer). The maximum harmonic order that can be generated depends on gas species. The generated harmonics propagate embedded in the residual laser beam as shown in Fig. 1.1.

Traditional low-order laser harmonic generation is done in solid materials such as crystals, where large fractions of laser pulses can be converted into second or third harmonic light. In contrast, HHG in crystals is not possible because the generated EUV light would be absorbed in the crystal. Furthermore, any solid will be severely damaged by the high intensities required to achieve HHG. Low-density gases, on the other hand, are less opaque in the EUV and are self healing after each laser shot. Atoms with high ionization energies are able to produce the highest harmonic orders because they withstand stronger laser fields before ionizing. Since noble gases have the highest ionization energies, they are good HHG targets. Helium and neon have the highest ionization potentials at $24.6 \mathrm{eV}$ and $21.6 \mathrm{eV}$, respectively, and consequentially are often employed to produce the highest possible harmonic orders. 


\subsubsection{Conversion Efficiency}

Conversion efficiency is often used to compare the effectiveness of different schemes for harmonic generation employed by different research groups. Conversion efficiency is the ratio of input energy of the generating laser to the output energy contained within the individual harmonics. The challenge is to achieve the highest possible conversion efficiencies for the highest possible harmonic orders to make useful sources of EUV light.

Rundquist et al. observed conversion efficiencies $>10^{-6}$ for harmonic orders 23 31 , yielding $>0.2 \mathrm{~nJ}$ of energy per harmonic order generated with $150 \mu \mathrm{J}$ laser pulses [8]. In their research they used a hollow capillary tube filled with argon, which allowed for good phase-matching. The tube guides the laser and allows for good phase-matching. Tamaki et al. reported a similar conversion efficiency of $10^{-6}$ for higher orders, around the $49^{\text {th }}$, generated in a neon-filled gas cell [9]. Takahashi et al. demonstrated a conversion efficiency of $1.5 \times 10^{-5}$ for the generation of the $27^{\text {th }}$ harmonic in an argon-filled cell [10]. In this case, a $20 \mathrm{~mJ}$ pulse energy produced $0.3 \mu \mathrm{J}$ energies for individual harmonics. More recently they demonstrated $5 \times 10^{-7}$ conversion efficiency for harmonics up to the $59^{\text {th }}$ order generated in neon, resulting in $25 \mathrm{~nJ}$ of individual harmonic energies from $50 \mathrm{~mJ}$ pulse energies [11]. They also reported record conversion efficiencies above $10^{-4}$ for lower order harmonics generated in xenon [12].

The brightness of high harmonics depends on a complex interplay of several factors, which include the parameters of the laser, gas species, phase matching, and re-absorption of the harmonics in the very gas used to generate them. Hence, efforts are taken to optimize each of these parameters to improve the generated signal. 


\subsection{Phase-Matching}

In order to have strong harmonic emission, the generating laser light and the created harmonics must travel in phase for a coherent buildup of energy. The harmonics generated upstream in the medium need to be in phase with new harmonics generated downstream. The coherence length, or a single phase zone, is the length over which the harmonic phases are matched. Outside of a coherence length, new harmonic production joins out of phase with previously generated harmonic light, causing destructive interference and less overall signal.

Phase mismatches can cause severe destructive interference for harmonic light emerging from different locations in the laser focus. There are at least three sources of phase mismatch: geometrical phase mismatch due to diffraction, frequency-dependent index of refraction, and intensity-dependent variations in the phases of the harmonics as they are emitted.

Geometrical phase mismatch is due primarily to the Gouy shift, which is a phase change of $\pi$ that all light beams experience as they go through a focus. The harmonics are generated with a phase $q$ times that of the laser, where $q$ is the harmonic order. This means that even though the laser experiences a phase shift of only $\pi$ as it goes through the focus, the emission of the $q^{\text {th }}$ harmonic is associated with a phase that ranges through $q \pi$. The phase variation in the emission pattern of the high harmonics is given by

$$
\phi_{\mathrm{Harm}}=q \phi_{\mathrm{Gouy}}=q \tan ^{-1}\left(\frac{z}{z_{0}}\right) .
$$

The Rayleigh range, $z_{0}=\pi w_{0}^{2} / \lambda$, is a parameter that depends on the laser beam waist $w_{0}$ and wavelength $\lambda[13]$. To find the coherence length, we take the derivative of the harmonic emission phase $\phi_{\text {Harm }}$ with respect to the propagation distance $z$ :

$$
\frac{\Delta \phi_{\text {Harm }}}{\Delta z}=\frac{q}{z_{0}\left[1+\left(\frac{z}{z_{0}}\right)^{2}\right]} .
$$




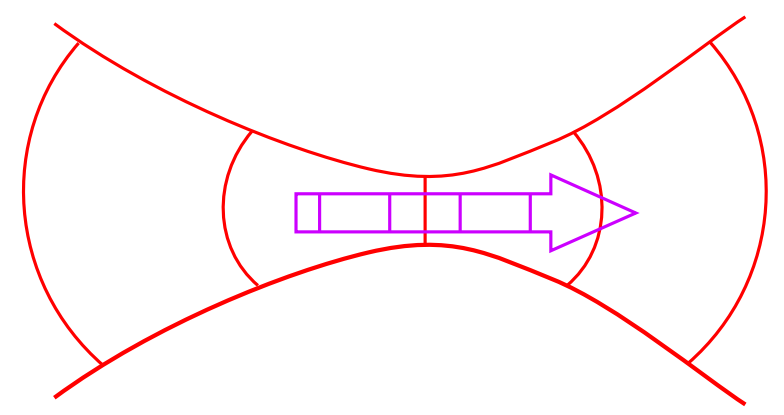

Figure 1.2 Depiction of how the harmonics(purple) diffract more slowly than the laser beam(red) as they travel through the focus.

Since we are interested in the region near the focus, we can set $z=0$ and solve for the $\Delta z$ that achieves a phase shift of $\Delta \phi_{\text {Harm }}=\pi$. This gives

$$
\Delta z=\frac{\pi z_{0}}{q}
$$

as an estimate for the coherence length of individual harmonics. For a typical Rayleigh range of one centimeter, the size of one phase zone $\Delta z$ (how far a harmonic can travel before it is out of phase by $\pi$ with additional generation) at the focus due to the Gouy shift varies between 300-700 $\mu \mathrm{m}$ for harmonic orders 49-99. This severe restriction on the coherence length is most pronounced for the highest harmonic orders. Fig. 1.2 depicts the wave fronts for the laser beam and a particular generated harmonic order. Because the wavelengths of the harmonics are much shorter than the laser wavelength, the harmonics can be thought of as plane waves since the rate of diffraction is so slow.

All media have an index of refraction that depend on the frequency of the propagating light. This causes the laser and the generated harmonics to travel at different speeds, which produces phase mismatches. Generally, this effect is small in comparison to the geometrical mismatch, but the process of HHG partially ionizes the gas, which creates a plasma. The associated difference in index for the laser versus the harmonics can lead to serious phase mismatches. The index of refraction in a plasma 
is given by

$$
n_{\text {plasma }}=\sqrt{1-\frac{N e^{2} \lambda^{2}}{4 \epsilon_{0} m \pi^{2} c^{2}}} \approx 1-\frac{N e^{2} \lambda^{2}}{8 \epsilon_{0} m \pi^{2} c^{2}},
$$

where $N$ is the number of free electrons per volume, $\epsilon_{0}$ is the permittivity of free space, $\lambda$ is the wavelength of the light, $e$ is the charge of an electron, and $m$ is the mass of an electron [13]. The plasma index effectively speeds up the laser pulse relative to the harmonic light, which because of much shorter wavelengths, has an index equal to vacuum for practical purposes. This gives rise to a coherence length (due to the plasma) of

$$
\Delta z=\frac{\lambda}{2 q\left(n_{\text {plasma }}-1\right)}
$$

We find that phase zones due to plasma index for the $49^{\text {th }}$ harmonic are about $100 \mu \mathrm{m}$ long, assuming 1\% ionization and 80 torr gas pressure. This phase mismatch has the same sign as that arising from the Gouy shift, which compounds the problem.

Another source of phase differences in generated harmonics arises due to spatial variations in laser intensity. Atoms located at different positions can emit harmonics with different phases, depending on the intensity they experience. The laser intensity can vary significantly both axially and radially within the gas medium.

\subsection{Phase Matching Techniques}

Much effort has been made to achieve favorable phase matching conditions for HHG. The most straight-forward method of minimizing geometrical phase mismatches is to use a narrow gas jet. The gas distribution produced by a typical gas jet can be as small as $300 \mu \mathrm{m}[14]$.

Milchberg et al. at the University of Maryland, [15] proposed that a plasma fiber waveguide can achieve phase matching of HHG over extended interaction lengths because the plasma waveguide mode structure is independent of wavelength. They re- 
ported guided pulses for distances $2.2 \mathrm{~cm}(\sim 70$ Rayleigh ranges) at intensities greater than $10^{14} \mathrm{~W} / \mathrm{cm}^{2}$, which are sufficient to generate HHG.

Durfee et al. investigated the phase matching of HHG in a gas-filled capillary waveguide [16]. A three-segmented capillary tube was used for their experiments, which allowed them to produce a constant pressure region within the center section $(3 \mathrm{~cm})$, while the end sections $(1.6 \mathrm{~cm})$ exited to vacuum. Intensities as high as $10^{14} \mathrm{~W} / \mathrm{cm}^{2}$ were achieved in the center capillary section. They reported that the more dispersive the gas, the lower the optimum pressure necessary for phase matching. By controlling the gas species, ionization level, and gas pressure, phase matching was achieved over several centimeters in xeon, krypton, argon, and hydrogen gases.

Tamaki et al. also used a gas-filled hollow fiber for HHG [17]. The guided region was filled with 20 torr of argon. The high-order harmonic spectrum emitted from the fiber showed an intensity distribution significantly different from that in free space. By comparing the two harmonic distributions, they observed more than a hundred times enhancement around the $25^{\text {th }}$ harmonic with the hollow fiber. This enhancement is attributed to macroscopic phase matching achieved in the hollow fiber.

Tamaki et al. demonstrated HHG in a neon-filled gas cell at 50 torr [9]. They observed a narrowing of the divergence angle of the transmitted beam, which they cited as evidence for laser filamentation. This was associated with enhancements of harmonic production up to 40 times for the $49^{\text {th }}$ harmonic, corresponding to conversion efficiencies of $10^{-6}$. Their laser pulses were generated by a Ti:sapphire laser, producing $100 \mathrm{fs}$ pulses with $5 \mathrm{~mJ}$ energies at a $10 \mathrm{~Hz}$ repetition rate. They used a gas cell with two $300 \mu \mathrm{m}$ diameter pinholes, isolating the gas from the vacuum chamber. Pinholes were used to transmit the focusing laser beam into and out of the cell. The propagation distance of the beam through the gas cell could be varied from 1-7 mm.

Tosa et al. [18] focused a $35 \mathrm{fs}, 10-20 \mathrm{~mJ}$ pulsed laser into a xenon-filled gas 
cell which had a $1.5 \mathrm{~mm}$ pinhole on each end surface, isolating the vacuum and gas-filled regions. They calculated the radial profile of the intensity, which they predicted should become nearly top-hat with a $250 \mu \mathrm{m}$ radius based on their model. Their modeling suggested that the intensity of the laser remained nearly constant throughout the cell, a characteristic of filamentation [19].

Kim et al. subsequently reported enhancements to harmonic generation associated with radial-flattening of the intensity and the existence of a long plasma streak [20]. A neon gas jet was used to generate harmonics up to the $61^{\text {st }}$ order in neon. The position of the gas jet was moved along the beam path in the focal region. The observed plasma streak was longest and maintained a uniform column shape when the gas jet was positioned $18 \mathrm{~mm}$ before the focus. The intensity profile at this position exhibited the top-hat profile.

\subsection{Filamentation}

The principal effects responsible for laser filamentation are well understood. Filamentation results predominantly from a dynamic competition between the optical Kerr effect and multiphoton ionization. The optical Kerr effect is responsible for selffocusing, while multiphoton ionization causes defocusing through dispersion. Multiphoton ionization occurs when the beam intensity reaches a sufficiently high value.

The index of refraction for a nonlinear medium can be written as:

$$
n=n_{0}+n_{2} I
$$

where $n_{2}$ is the optical Kerr constant. The Kerr constant for an atmosphere of helium is $3.5 \times 10^{-21} \mathrm{~cm}^{2} / \mathrm{W}$ compared to the value of air of $5.0 \times 10^{-19} \mathrm{~cm}^{2} / \mathrm{W}$ [21]. The Kerr effect is only observed at high intensities, similar to those required for harmonic

generation. Our laser beam setup can achieve intensities up to $10^{16} \mathrm{~W} / \mathrm{cm}^{2}$. Filaments 
in air have been observed to maintain a beam radius of $100 \mu \mathrm{m}$ for distances of over a hundred meters, well beyond the Rayleigh range [22] . Intensity-dependent losses eventually deplete the energy in the channel and destabilize the process.

The interplay between self-focusing and ionization is a highly dynamic process, with spikes of ionization occurring whenever the beam starts collapsing again [23]. Energy losses during filamentation are minimal for two reasons: (a) the intensity is always maintained at or below the value corresponding to ionization and (b) the short pulse duration prevents significant pulse attenuation through its interaction with the generated plasma [24].

Braun et al. in 1995, reported the first observation of femtosecond lasers generating filaments in air with intensities of $7 \times 10^{13} \mathrm{~W} / \mathrm{cm}^{2}$ propagating distances greater than $20 \mathrm{~m}$ [25]. They used a Ti:sapphire laser with pulse duration of $200 \mathrm{fs}$ and pulse energies as high as $50 \mathrm{~mJ}$. As the pulse energies were increased from $5 \mathrm{~mJ}$ to $20 \mathrm{~mJ}$ the measured beam diameter (FWHM) decreased from $6 \mathrm{~mm}$ to $80 \mu \mathrm{m}$, showing direct evidence of filamentation. Although the distances involved in high harmonic generation are much shorter than for traditional filamentation experiments, the fundamental dynamics may well be similar.

\subsection{Previous High-Harmonic Work at BYU}

Harmonic generation has been studied here at BYU for the past decade in the group of Dr. Peatross. Recently, a masters student (Julia Sutherland) probed the focal region with counter-propagating pulses to determine where harmonics are produced [26]. Sutherland's work revealed that harmonics are phase matched over a much greater distance than expected, so much so that in most cases, only a single phase zone near the exit foil was found. The harmonics were phase matched well enough 

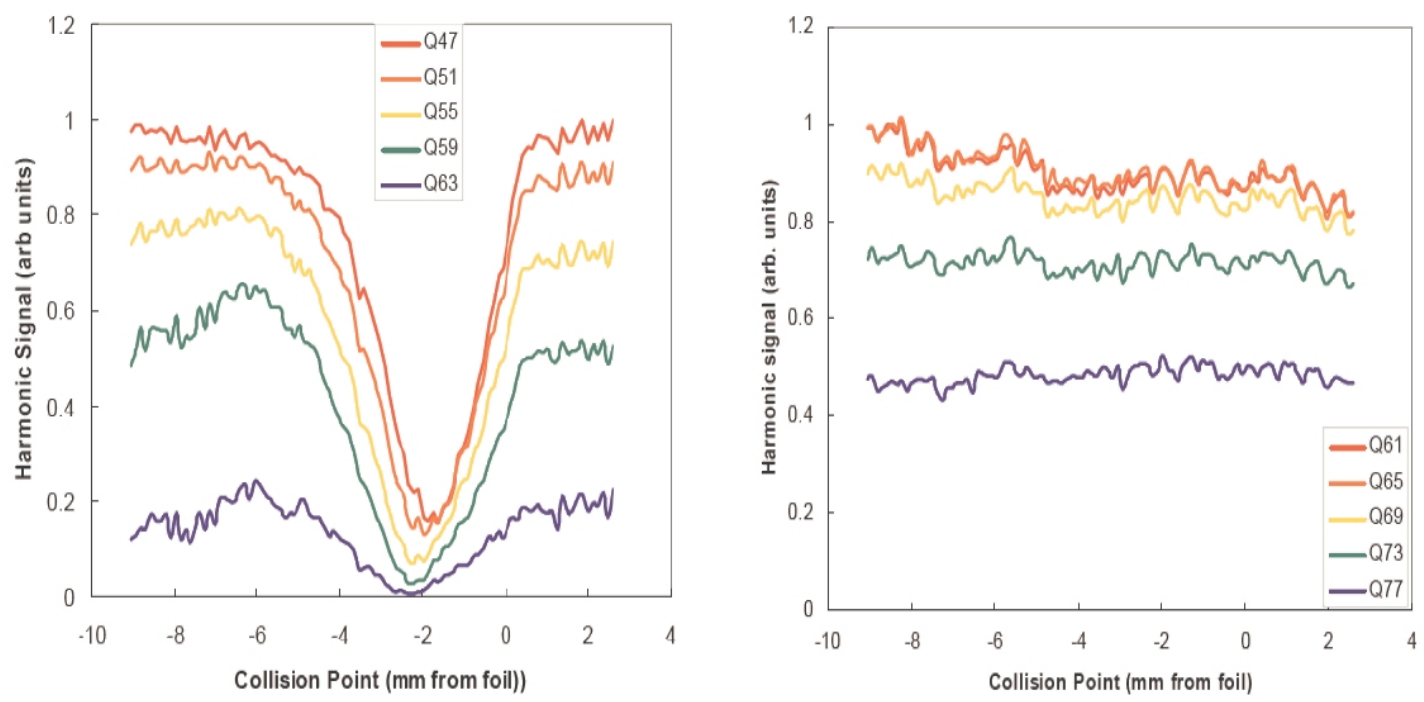

Figure 1.3 A counter-propagating pulse was able to disrupt phase matching in neon(left) when it collided with the generating pulse near the exit foil. No effect was observed in helium(right) [26].

that the re-absorption limit was reached in the case of neon, but not helium, see Fig. 1.3. The re-absorption limit is a point where increasing the interaction length or increasing the gas pressure in the generating medium gives diminishing returns. This was quite unexpected, since geometrical phase mismatches alone were expected to have produced many opposing phase zones within the HHG region.

Sutherland's work provided strong evidence for the existence of filamentation, as suggested by Tamaki et al. [9]. Filamentation might give much longer phase zones due to prevention of natural diffraction. In this thesis, we report on direct measurements of laser-beam evolution near the focus inside the gas cell under conditions ideal for harmonic generation.

It is well known that harmonic output can be severely limited by re-absorption in the generating medium. Eric Christensen, a previous undergraduate student in our group, and Julia Sutherland studied the effect of re-absorption in HHG [27]. They showed that the re-absorption limit was reached in neon but not in helium. In neon 
the gas pressure was optimized at 55 torr, meaning that the re-absorption depth was only about $2 \mathrm{~mm}$. On the other hand, they showed that in helium the harmonics would need to be phase-matched over a distance of $10 \mathrm{~mm}$ in order to reach the re-absorption limit for a gas pressure of 120 torr. Indeed, this is consistent with Fig. 1.3 which shows that the coherence length in helium is far longer than $2 \mathrm{~mm}$ (the distance over which it was possible to suppress harmonic production with the counter-propagating light).

\subsection{Outline of Experiments}

In order to directly observe and measure possible filamentation in our laser focus, we designed and built an apparatus to image the laser focus (at full power) onto a CCD camera. The optimal conditions (i.e. laser power, gas pressure, aperture diameter, etc.) for HHG were used for the experiment. The exit foil of the gas cell was moved many centimeters as the evolution of the beam was recorded. Similar images were taken with an evacuated gas cell for comparison.

The end of a small fiber optic coupled to a spectrometer was swept through the imaged focus to measure any radial variations in the focal spectrum as well as overall shifts in the spectrum compared to vacuum. Only minor radial variations were seen in the focus. The spectrum for the helium-filled cell in the center of the beam was blueshifted by about $4 \mathrm{~nm}$ compared to no shift in the evacuated cell. This is a modest shift compared to the spectral width of the laser which is approximately $35 \mathrm{~nm}$ wide.

Additionally, the absolute energy of the individual harmonics was determined for harmonics ranging from the $41^{\text {st }}$ to the $91^{\text {st }}$. The measured energy per individual harmonic order is $\sim 1 \mathrm{~nJ}$, which corresponds to a conversion efficiency of $2 \times 10^{-7}$. This is comparable to what other groups have reported. The higher conversion efficiencies 
reported by Takahashi et al. mentioned earlier are for lower harmonic orders than we characterized.

\subsection{Thesis Overview}

The experimental setup used for this work is discussed in detail in chapter 2. Section 2.1 describes the laser system, including how the laser pulses are delivered to the gas cell. Section 2.2 provides an overview of how the high harmonics are generated and detected. Section 2.3 describes the imaging apparatus used to measure the laser focus during the harmonic generation process. Section 2.4 explains the process of ensuring proper alignment of the imaging apparatus. The final section lists the parameters that are varied to optimize harmonic generation.

Chapter 3 reports the experimental results that characterize the evolution of the laser in the focus. The first section presents scans of the focal images for various exitfoil positions. Corresponding images were also recorded with the gas cell evacuated for comparison. Section 3.2 presents scans of the focal images for the same exit foil positions but with an aperture located $145 \mathrm{~cm}$ upstream of the focusing mirror being partially closed. Section 3.3 shows comparisons between the beam profile obtained for propagation in helium and propagation in vacuum. The final section in chapter three contains the beam width (FWHM) measurements of our beam in helium and vacuum.

Chapter 4 presents auxiliary measurements performed on the laser beam and generated harmonics. The first section discusses pulse-duration measurements. Section 4.2 reports measurements of the spectral content and the influence of the interaction with the gas cell on the spectrum. Section 4.3 outlines the method used to measure the energy of individual harmonic beams as well as gives the conversion 
efficiency for our semi-infinite gas cell configuration. Chapter 5 discusses the results and offers an outlook for future work. 


\section{Chapter 2}

\section{Experimental Setup and Procedures}

\subsection{Laser System}

For the experiments presented in this thesis we used a Ti:sapphire laser which delivers about $10 \mathrm{~mJ}$ of energy per shot at a repetition rate of $10 \mathrm{~Hz}$. The laser pulses are centered around $800 \mathrm{~nm}$ with bandwidth of $35 \mathrm{~nm}$ (FWHM). After amplification, the laser beam is expanded in a Galilean telescope to a diameter of $11 \mathrm{~mm}$. Fig. 2.1 shows a near-field image of the laser beam $35 \mathrm{~cm}$ before the focus, which shows nonuniform structure, which is characteristic of many high-power laser systems. The laser is focused using a concave mirror $(f=100 \mathrm{~cm})$. The $\mathrm{f} / 91$ focusing predicts a focal-spot radius of $w_{0}=46 \mu \mathrm{m}$, compared to the measured value of $w_{0}=58 \mu \mathrm{m}$, as shown in Fig. 2.2. This indicates that the laser focuses to 1.25 times the diffraction limit, owing to imperfections in the beam.

Prior to the focusing mirror, the laser pulses are temporally compressed using parallel gold-coated gratings (1200 lines per millimeter). The pulse duration, measured 


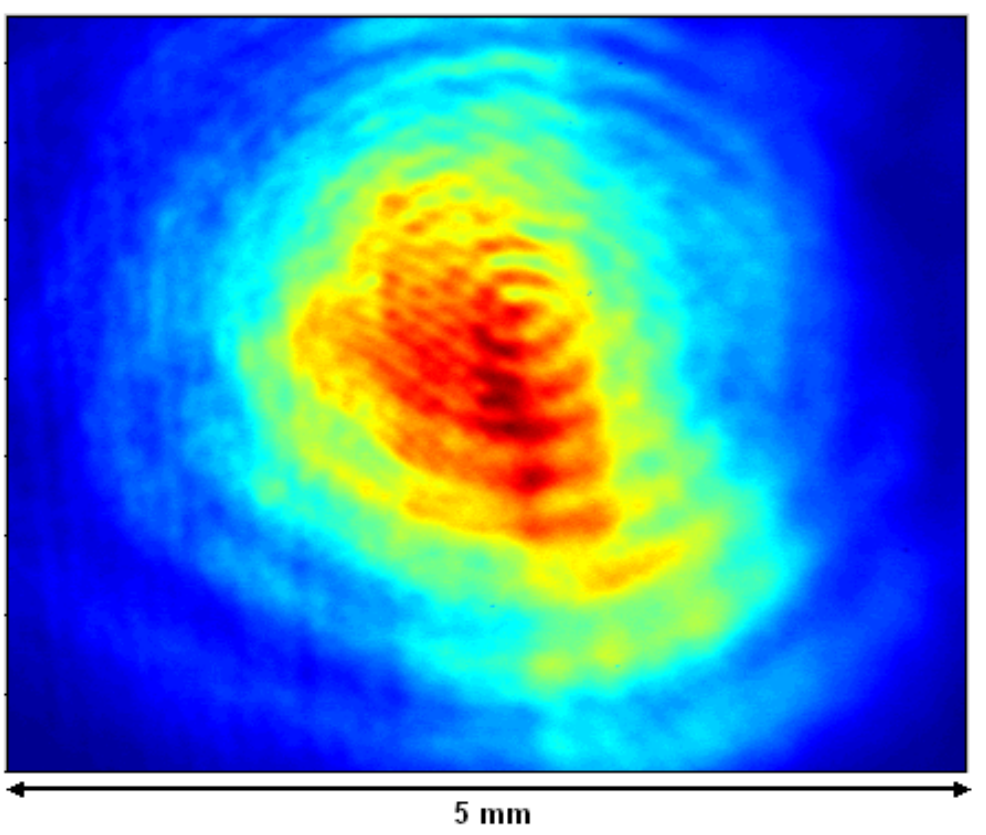

Figure 2.1 Direct measurement of laser beam $35 \mathrm{~cm}$ before focus with aperture open showing non-uniformity of the structure.
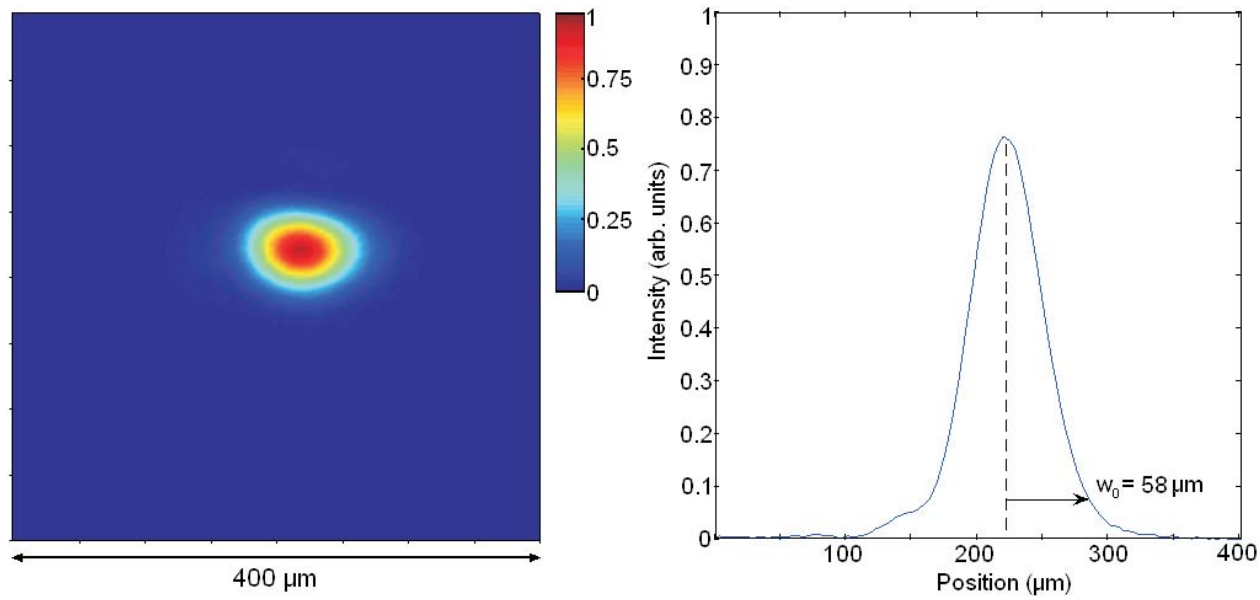

Figure 2.2 Laser-beam profile at the beam focus, which has a measured focal-spot radius of $58 \mu \mathrm{m}, 1.25$ times the diffraction limit. 


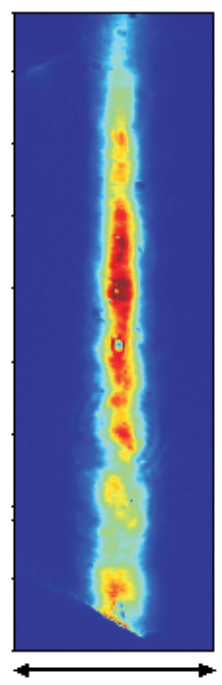

$1.5 \mathrm{~mm}$

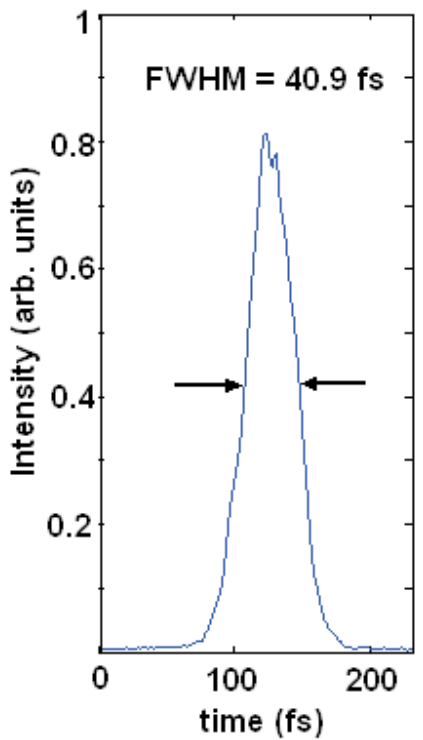

Figure 2.3 Auto-correlation trace of the 30 fs laser pulse. The trace is $\sqrt{2}$ wider than an individual pulse, assuming a Gaussian profile.

by autocorrelation, was determined to be about 30 fs. The autocorrelation trace is shown in Fig. 2.3, which is produced by splitting a pulse into to identical ones and mixing them in a nonlinear crystal. The interaction of the two beams in the crystal maps the temporal profile onto a spatial image. The width of the autocorrelation trace is $\sqrt{2}$ times wider than the original pulse, assuming a Guassian temporal profile.

The intensity of the focused laser pulse is estimated to be $I_{o} \simeq E\left(\frac{\pi w_{o}^{2}}{2} T_{\mathrm{FWHM}}\right)^{-1}$, where $E$ is the pulse energy, $\omega_{0}$ is the laser oscillation frequency, and $T_{\mathrm{FWHM}}$ is the FWHM of the time duration for the pulse. Our measured parameters gives $6 \times 10^{15} \mathrm{~W} / \mathrm{cm}^{2}$. This is more than sufficient to ionize helium, which readily ionizes at $1.5 \times 10^{15} \mathrm{~W} / \mathrm{cm}^{2}$. As will be further described, the intensity of our laser will drop from the measured value as it interacts with helium gas. A partially closed aperture used on the laser beam during the experiment also attenuates the peak intensity. 


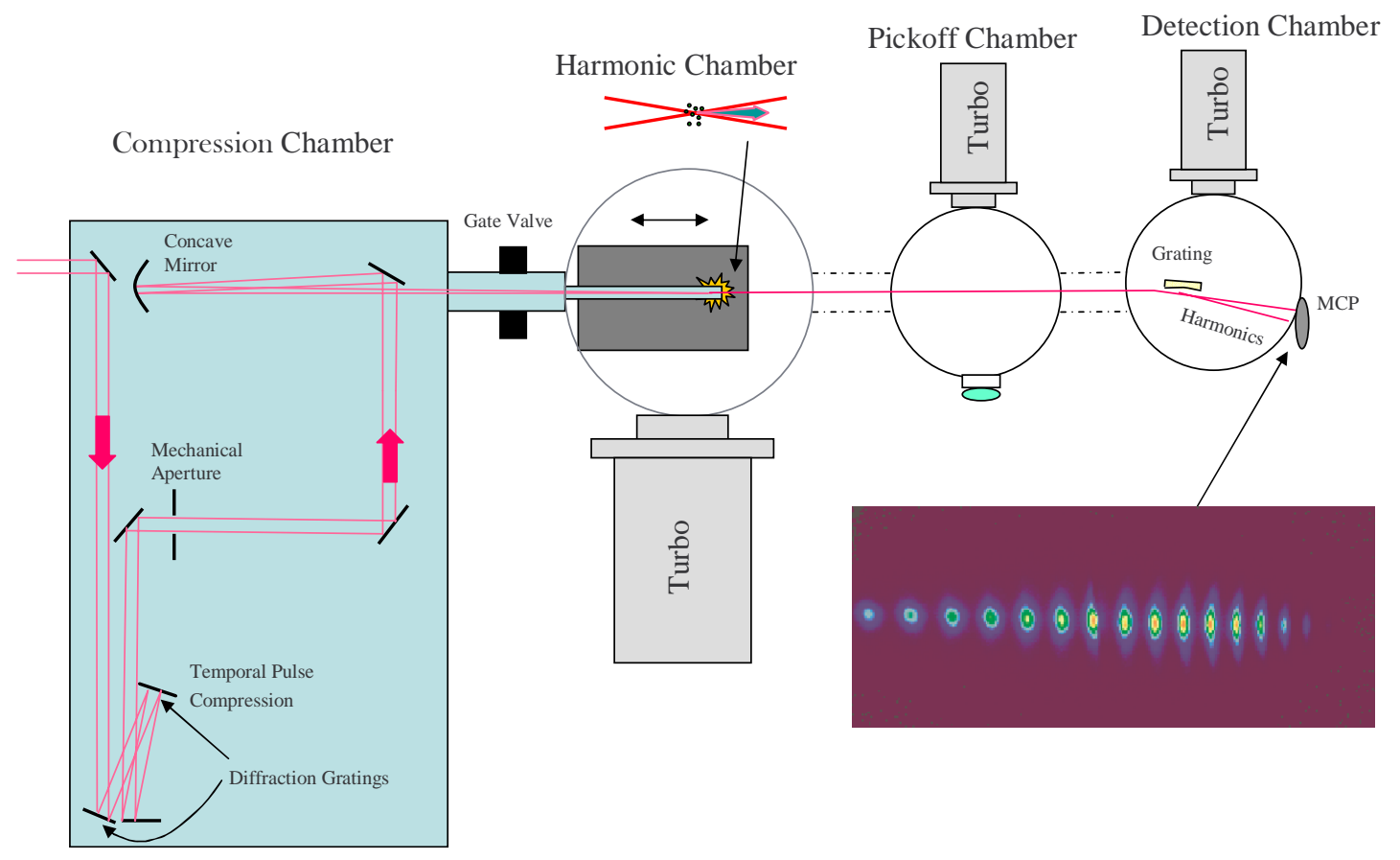

Figure 2.4 Experimental setup of chambers and laser pulse delivery.

\subsection{Experimental Setup}

The compression gratings and focusing mirror for the laser are contained in a large vacuum chamber that can be backfilled with helium or other gases. A schematic of the entire experimental setup is shown in Fig. 2.4. The gas-filled region extends to the laser focus where it abruptly ends as the laser passes through a pinhole into vacuum. The pinhole is created before each experimental run by using the laser to drill a hole through a 100- $\mu$ m-thick molybdenum foil. This automatically ensures that the pinhole is aligned to the laser beam.

We refer to the gas-filled region as a semi-infinite gas cell. High-order harmonics are generated in the focus of the laser beam, just before it exits into vacuum. The generated harmonics emerge embedded in the residual laser beam. The laser and the harmonic beams proceed together to the final chamber about one meter away. Small 


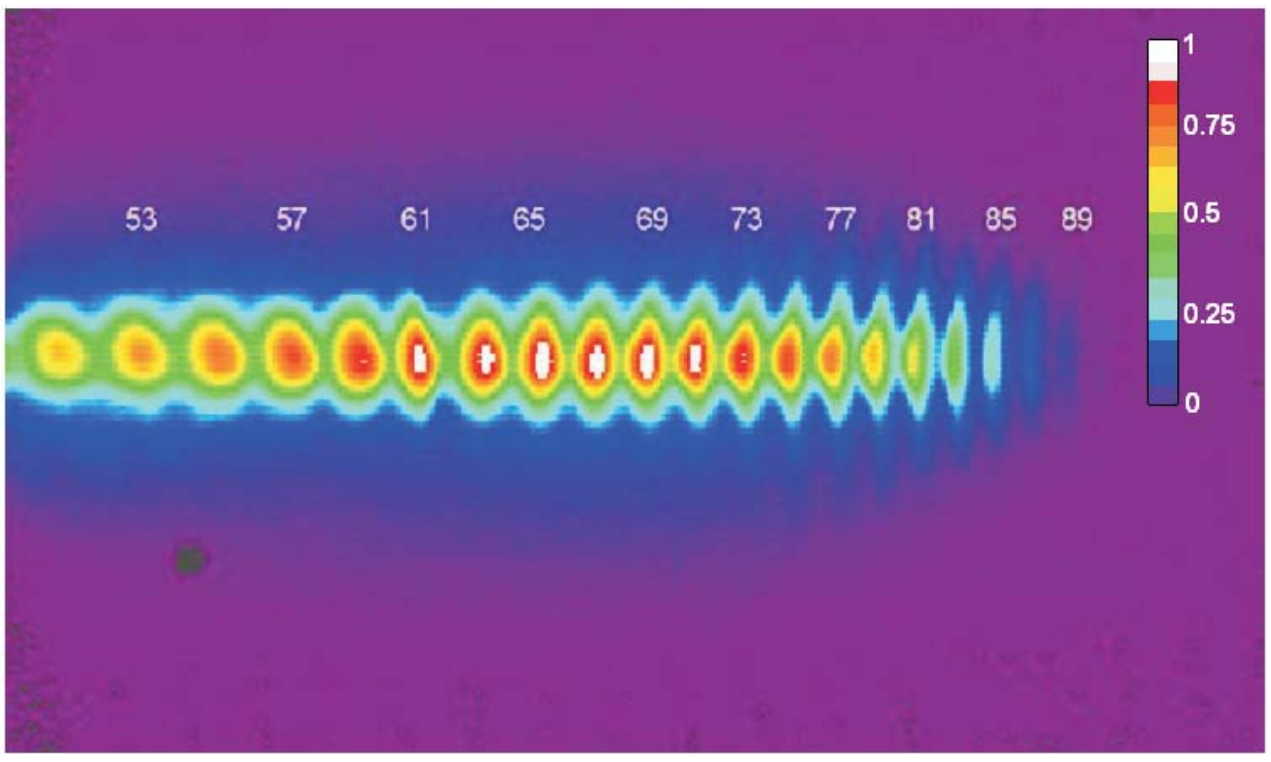

Figure 2.5 Harmonic orders 51 - 89 generated in 85 torr helium.

holes between the chambers allow the light to pass through from one to the next. The various chambers are connected to turbo pumps for differential pumping. This allows the pressure in the final chamber to be kept at $\sim 10^{-6}$ torr, which is necessary for proper function of the Micro-Channel-Plate (MCP) EUV detector.

A grazing-incidence diffraction grating (1200 lines/mm , $2 \mathrm{~m}$ radius of curvature) intercepts the beams of harmonics approximately $26 \mathrm{~cm}$ before the MCP. The grating separates each harmonic according to wavelength and focuses them onto the MCP. The harmonics are separated horizontally into sharp lines, which preserve their spatial profile in the vertical dimension. The MCP is coupled to a $30 \mathrm{~mm}$-diameter phosphor screen on which individual harmonic orders appear as vertical stripes. The harmonic images are captured with a CCD camera. Fig. 2.5 shows a typical image of harmonics, displayed in false color, which indicates intensity.

Earlier work by Julia Sutherland [26] showed that harmonic generation was dependent on the position of the gas-cell exit (molybdenum foil) relative to the laser 
beam focus. Foils are glued to glass tubes that can be inserted into a sealed sleeve. The sleeve is connected to the gas-filled chamber via a flexible bellows. The whole assembly sits on top of a translation stage that allows for movement along the axis of the laser (with an automated scan range of $5 \mathrm{~cm}$ ). The translation stage sits on tilt and rotation stages (for pitch and yaw adjustment) to ensure that the translation occurs parallel to the beam path. The alignment of the translation axis is discussed in the next section.

Sutherland also found that placing an aperture in the beam path before the focusing mirror enhances harmonic output. We placed a motorized aperture in the laser beam path $145 \mathrm{~cm}$ before the focusing mirror. A remote camera allows adjustment of the aperture diameter during experiments. Figs. 2.6 and 2.7 show images of the laser beam taken $36 \mathrm{~cm}$ before and at the laser focus, respectively, with the aperture partially closed to form a 9-mm-diameter circular hole. An Airy pattern appears at the focus with radius of $110 \mu \mathrm{m}$ to the dark ring, which agrees with prediction: $r=\frac{1.22 \lambda f}{D}=108 \mu \mathrm{m}$, where $f$ is the mirror focal length and $D$ is the aperture diameter.

During experiments, the large vacuum chamber is filled with approximately 80 torr of helium. This requires approximately 30 minutes, in order to pump out the large chamber and introduce the helium. A gate valve is used to isolate the gas-filled chamber from the subsequent chamber that houses the cell exit foil. This allows the foil to be changed without needing to re-pump down the large chamber.

\section{$2.3 \quad$ Imaging Apparatus}

A beam pick-off chamber is located between the harmonic-production chamber and the harmonic detection chamber as seen in Figure 2.8. The beam pick-off chamber 

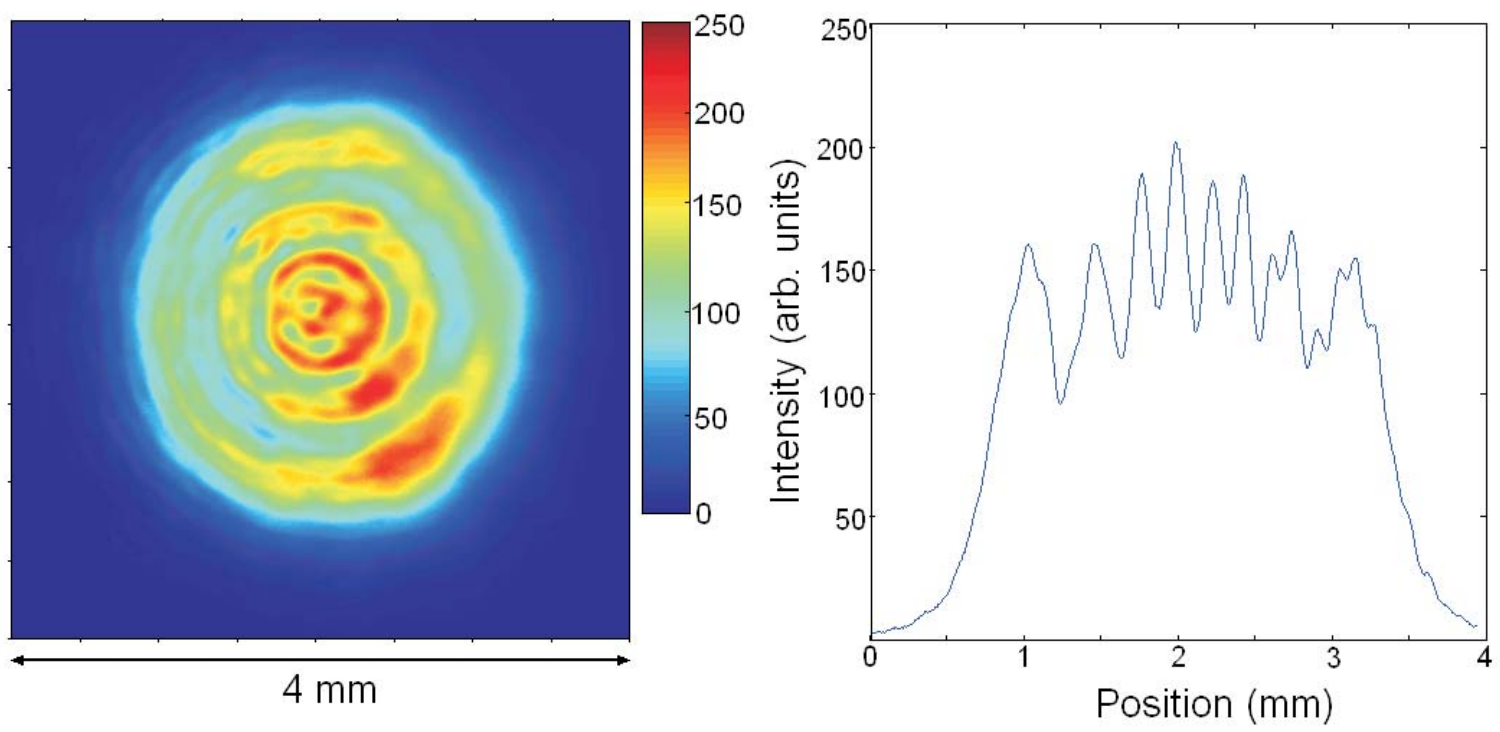

Figure 2.6 Near field image of laser beam $36 \mathrm{~cm}$ before focus with aperture diameter at $9 \mathrm{~mm}$.
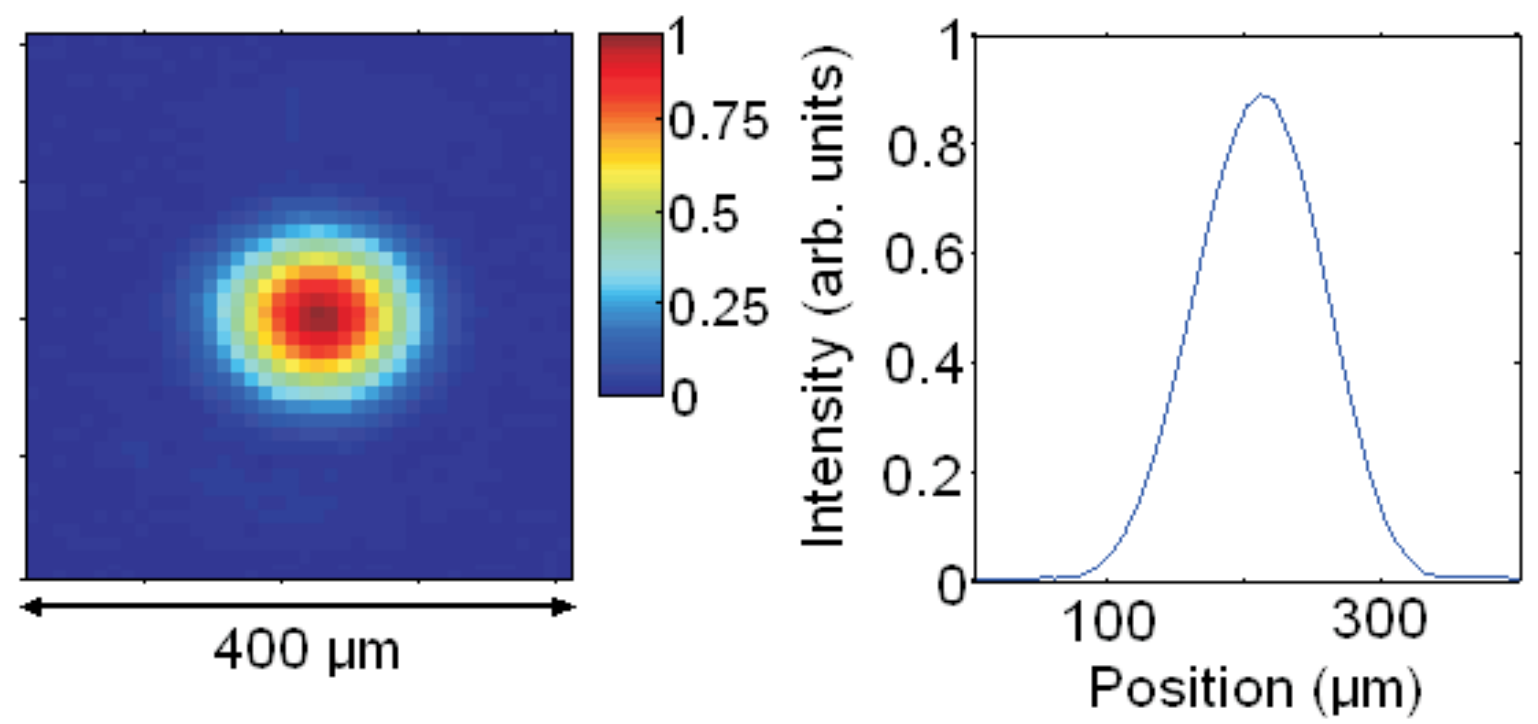

Figure 2.7 Near field image of laser beam at the focus with aperture diameter at $9 \mathrm{~mm}$. 


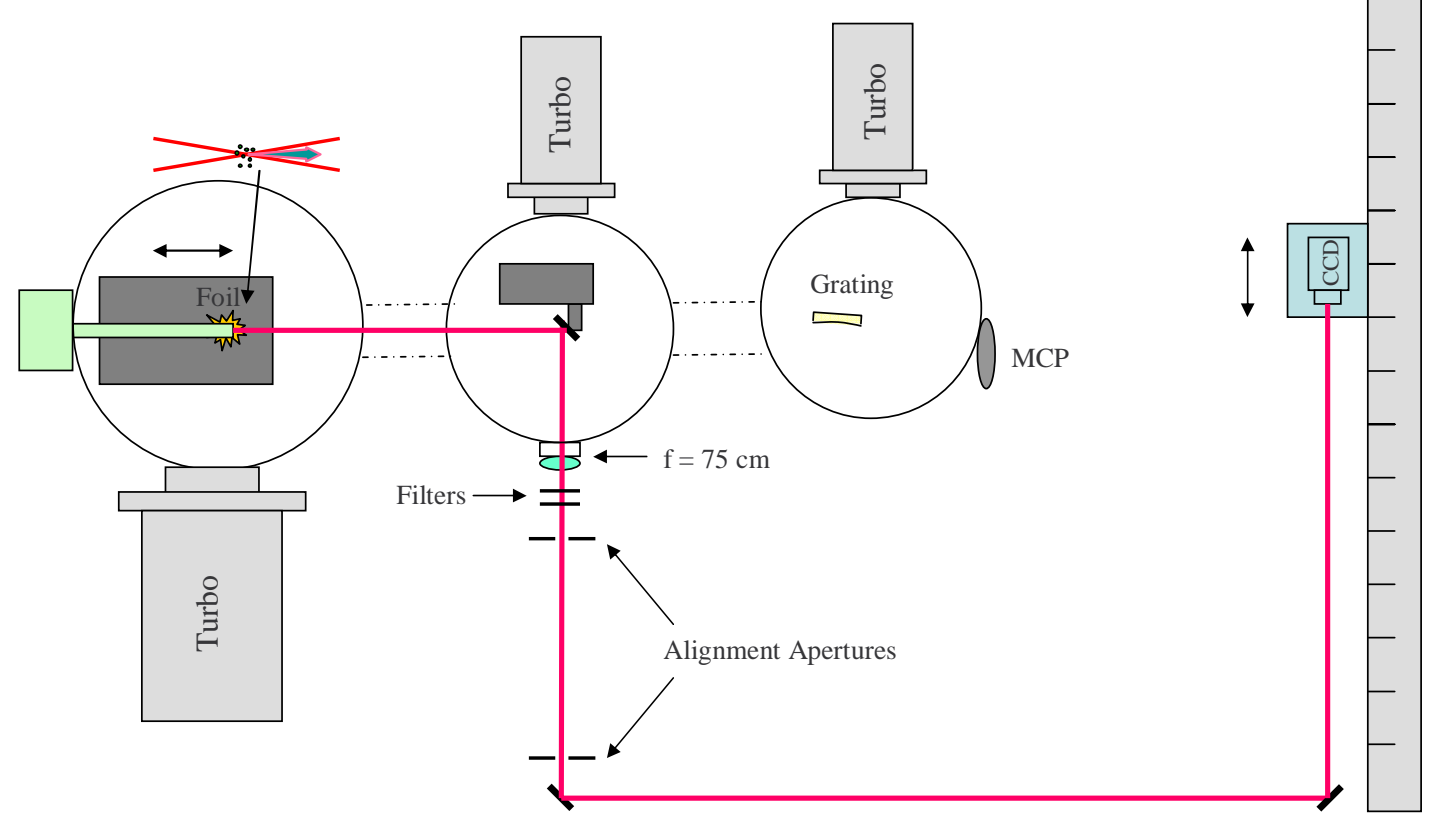

Figure 2.8 The apparatus used to image the laser focus onto a CCD camera.

accommodates an uncoated glass substrate attached to a vertically mounted translation stage. The uncoated glass surface (oriented at 45 degrees) reflects $2 \%$ of the p-polarized laser light. This attenuation saves a focusing lens (which also serves as a window for the laser to exit the chamber) from laser damage. The vertical translation stage can raise and lower the glass reflector in only a few seconds, without breaking vacuum, to allow harmonics to pass to the detection chamber, or to send the laser beam out to a camera for imaging of the laser focus. The deflected beam goes through a plano-convex lens $(f=75.5 \mathrm{~cm})$ as it exits the chamber. The laser beam is further attenuated with neutral-density filters and then reflected off two silver mirrors and onto a CCD camera as shown in Figure 2.8.

The distance from the typical gas-cell-foil position to the imaging lens is about $98 \mathrm{~cm}$. This produces an image of the foil position at $350 \mathrm{~cm}$ after the imaging lens, for a magnification of $3.5 \times$. The magnification varies as the foil position is varied 

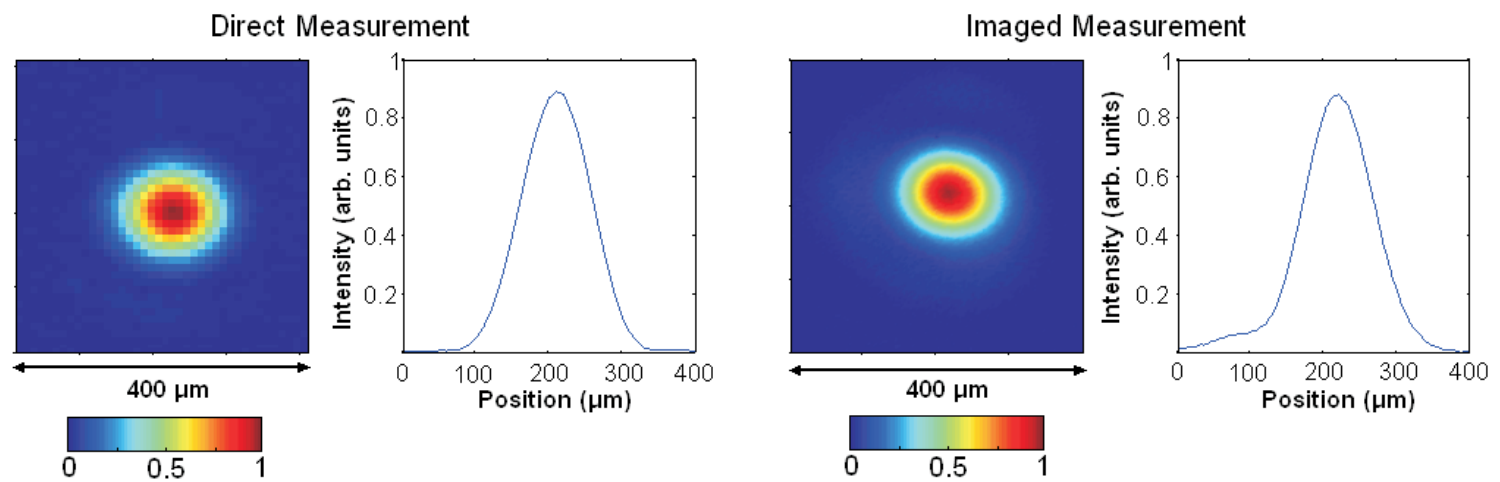

Figure 2.9 Direct measurement of the laser focus compared to the corresponding measurement using the imaging system (in vacuum).

and the camera is moved to keep it in focus. The appropriate imaging distance is determined via

$$
\frac{1}{f}=\frac{1}{p}+\frac{1}{q}
$$

where $f$ is the focal length of the imaging lens, $p$ is the distance from the exit foil to the imaging lens, and $q$ is the distance from the imaging lens to the CCD camera. An aluminum rail is bolted to the table to guide alignment of the CCD camera. The filters placed in the beam path protect the CCD camera from damage and allow for correct energy exposure.

To ensure the validity of the imaging apparatus we compare the beam focus in the near field to the imaged beam focus in vacuum, see Fig. 2.9. In both cases the aperture diameter is $9 \mathrm{~mm}$ and the focus diameters agree to within $5 \%$.

\subsection{Alignment of the Imaging Apparatus}

We depended on the imaging system to verify that the laser was aligned parallel to the gas-cell translation stage. Therefore, it was necessary to verify that the laser propagated parallel to the axis of the imaging lens. To ensure this, we first used a HeNe laser to mark the imaging-lens axis. Two apertures were placed outside of the 

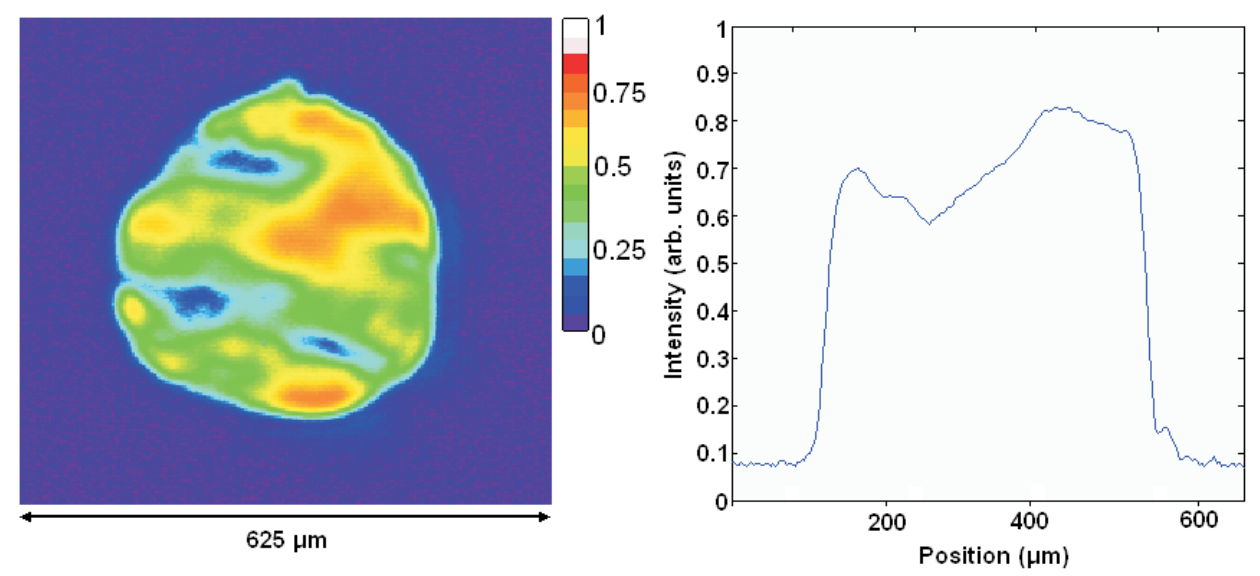

Figure 2.10 Image of the pinhole used for alignment of the gas cell translation stage.

pick-off chamber as seen in Fig. 2.8. We relied on ghost reflections from the front and back surfaces of the lens.

The Ti:sapphire laser was carefully aligned such that it passed through the two apertures. A molybdenum foil with a pre-drilled pinhole was then installed. A diffuser (scotch tape) was placed in the laser beam upstream from the foil to back-illuminate the pinhole. The tape also attenuated the laser beam, so very few filters were needed for appropriate camera exposure.

At the image plane of the foil, the camera sees a sharp image of the backilluminated pinhole as shown in Fig. 2.10. This allowed us to confirm that we were at the focus. This is crucial for our experiments, which require the capture of laser images at the foil plane as the foil is scanned.

To ensure that the translation stage under the foil moved parallel to the beam, we watched on the CCD camera to see if the image of the pinhole moved laterally as the stage was scanned. Adjustments were made to yaw and pitch until the pinhole image shifted laterally no more than about $40 \mu \mathrm{m}$ through the entire translation range $(5 \mathrm{~cm})$. This is small compared to the typical pinhole size of $400 \mu \mathrm{m}$ as seen in Fig. 2.10. 


\subsection{Optimizing Parameters for High Harmonic Pro- duction}

Previous work in our research group by Julia Sutherland showed that the diameter of the aperture placed upstream in the laserpath drastically affects the harmonic output [26]. We have since observed that the location of the aperture relative to the focusing mirror also affects the harmonic output signal. An optimal location was identified to be $1.5 \mathrm{~m}$ or more before the focusing mirror.

We investigated the relationship between foil location relative to the beam focus. Sutherland reported that the optimal location for the gas-cell exit foil in the presence of 100 torr helium is $9 \mathrm{~mm}$ before the focus [26]. We found the optimal position of the foil to be at best focus (i.e. where the laser focuses best under vacuum conditions).

We also adjusted gas pressure and pulse compression to optimize high harmonics. We found that 80 torr was the best gas pressure. Before the experiment, the compression gratings were optimized in air. Once the helium was introduced into the chamber the compression gratings could be adjusted to enhance the harmonics, but this is most likely just to get back to optimal compression, taking into account the difference of index of refraction between air and helium inside the pulse compression system. This is discussed more in section 4.1. 


\section{Chapter 3}

\section{Evolution of Laser Spatial Profile}

\subsection{Harmonic Generation at Different Gas-Cell Po- sitions}

As an intense laser pulse focuses within the gas cell, the interaction with the gas influences its propagation. The focus evolves through a complicated interplay between diffraction, nonlinear self-focusing, and dispersion through the rapid production of free electrons. Using the laser-beam imaging system described in section 2.3 together with the harmonic detection system described in section 2.2 , we recorded the evolution of the laser beam and the associated harmonic emission as we scanned the position of the gas-cell exit foil along the laser axis. The structure of the beam constantly changes as it propagates in the gas cell. For each foil position, the imaging system recorded the spatial profile of the laser in the plane of the exit foil.

Images of harmonic production for each of the exit foil positions were saved on the computer. The deflection mirror was then lowered and the beam was sent through the $\mathrm{f}=75.5 \mathrm{~cm}$ focusing lens and onto the imaging $\mathrm{CCD}$ camera. The exit foil was scanned through the focus region by moving the translation stage beneath the gas 
cell. The CCD camera was placed at the predetermined position so that the foil plane was in focus at the camera.

Figs. 3.1 and 3.2 show the harmonic emission and the measured laser profile for a range of foil-exit positions. The foil positions are referenced to the mirror focus $(100 \mathrm{~cm})$, which is where the laser focuses in the absence of gas. Negative numbers labeled in the figures correspond to positions before the focus, or closer to the focusing mirror. The gas cell (which includes the large first chamber) was backfilled with 80 torr helium. The removal of air and its replacement with helium affects the pulse compression by the grating pair, effectively changing the separation between the gratings. During the experiment, the grating was adjusted to optimize pulse temporal compression for harmonic production, which as shown in the next chapter, which takes place close to the shortest pulse duration available $(30 \mathrm{fs})$.

The images, which show the laser-beam profile over a scan distance of $5 \mathrm{~cm}$, reveal significant irregularity and structure. As is seen in the figures, the harmonics that are produced are relatively weak. In the next section, we demonstrate that partially closing an aperture on the laser beam upstream from the focusing mirror causes the harmonics to increase in brightness dramatically. At the same time the focal spot of the laser grows in size and becomes more regular.

\subsection{Apertured Laser Beam}

By partially closing the aperture positioned $145 \mathrm{~cm}$ before the focusing mirror, the harmonic output was enhanced and the laser beam profile became smoother than in the open aperture case. A comparison of the intensity of the harmonics for the open aperture and partially closed aperture is shown in Fig. 3.3.

It was determined that a $9 \mathrm{~mm}$ diameter aperture opening resulted in the brightest 

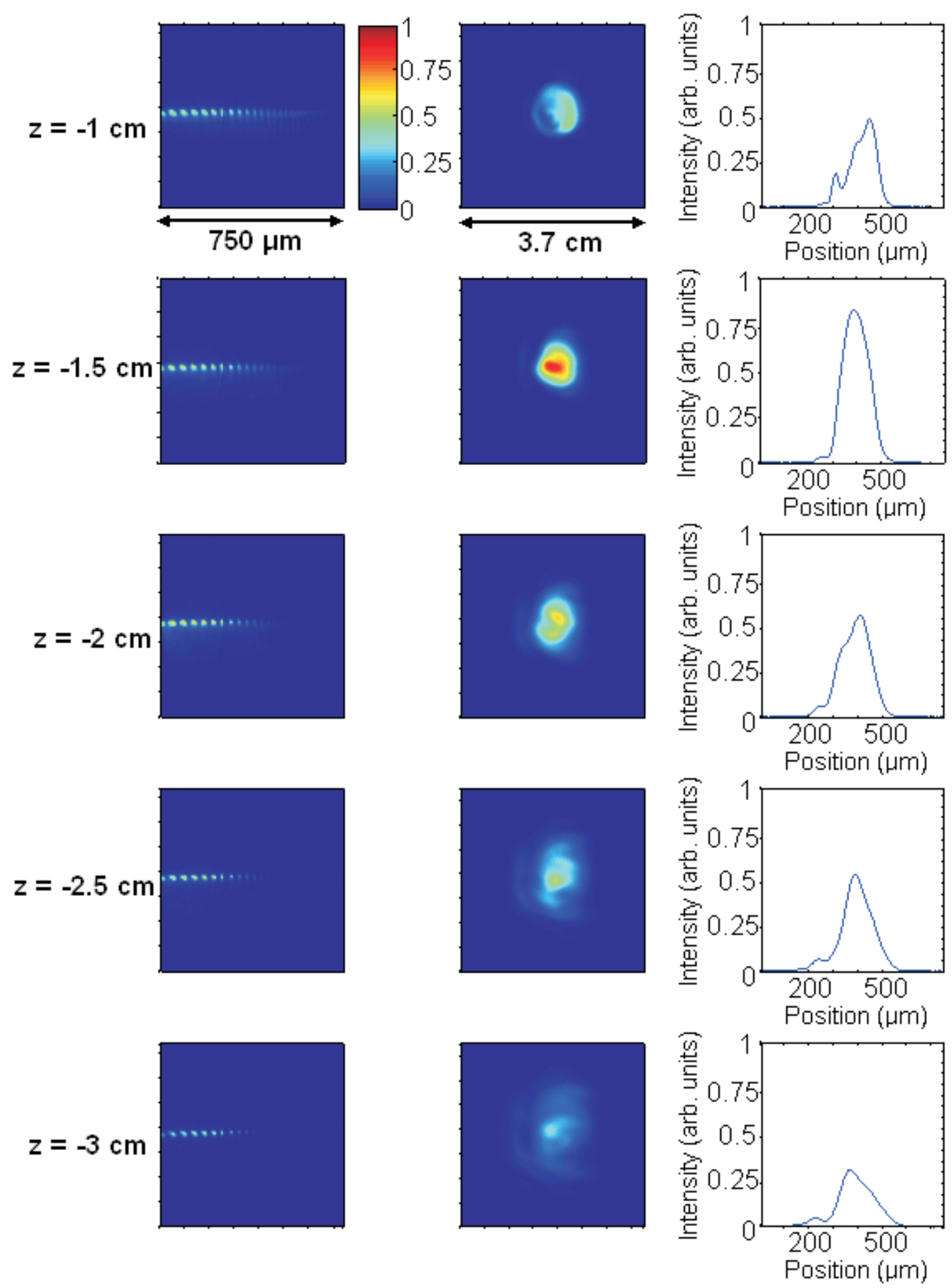

Figure 3.1 Harmonics generated at various foil positions with the laserbeam aperture open, along with beam profile images. 


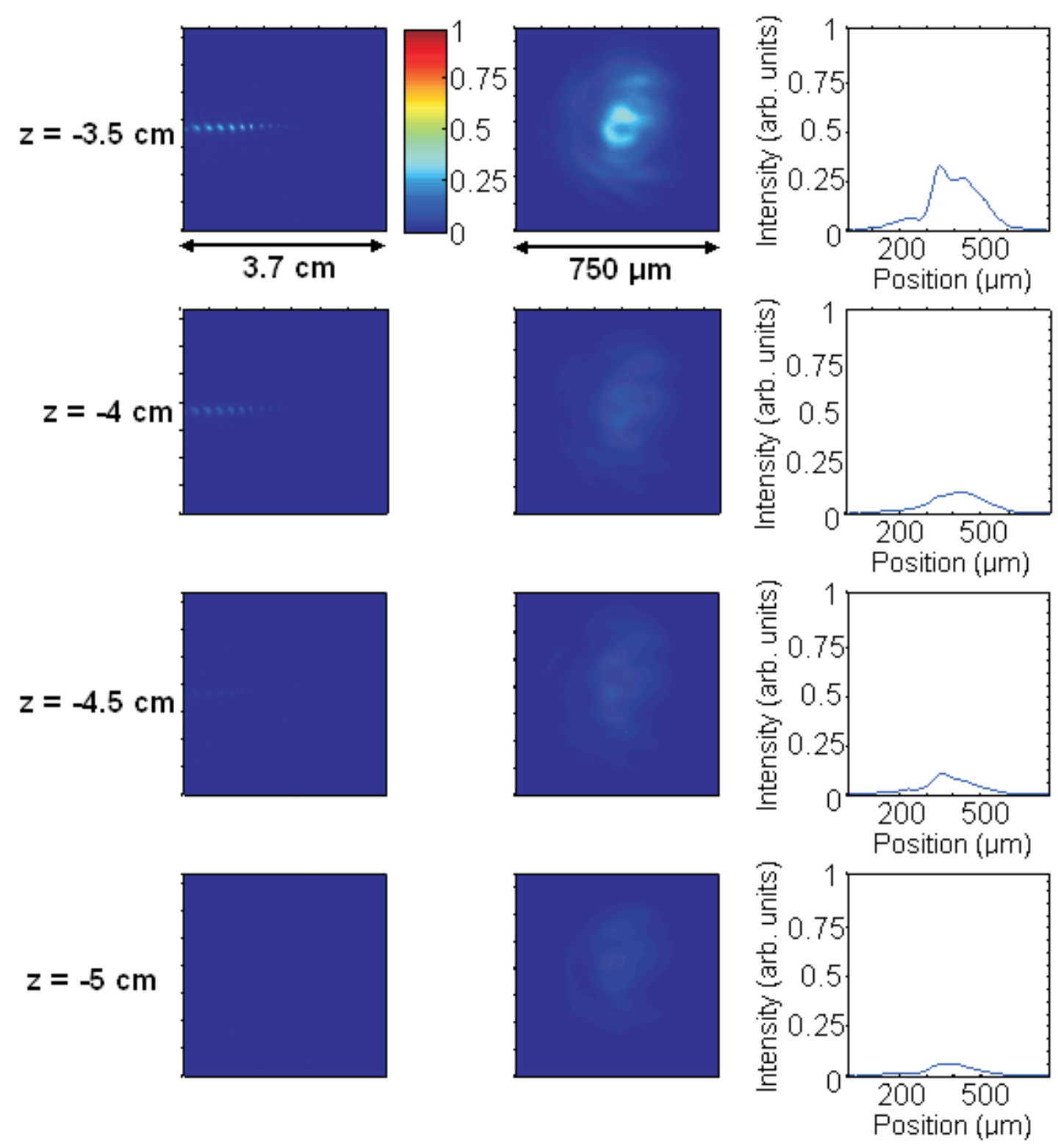

Figure 3.2 Harmonics generated at various foil positions with the laserbeam aperture open, along with beam profile images. 

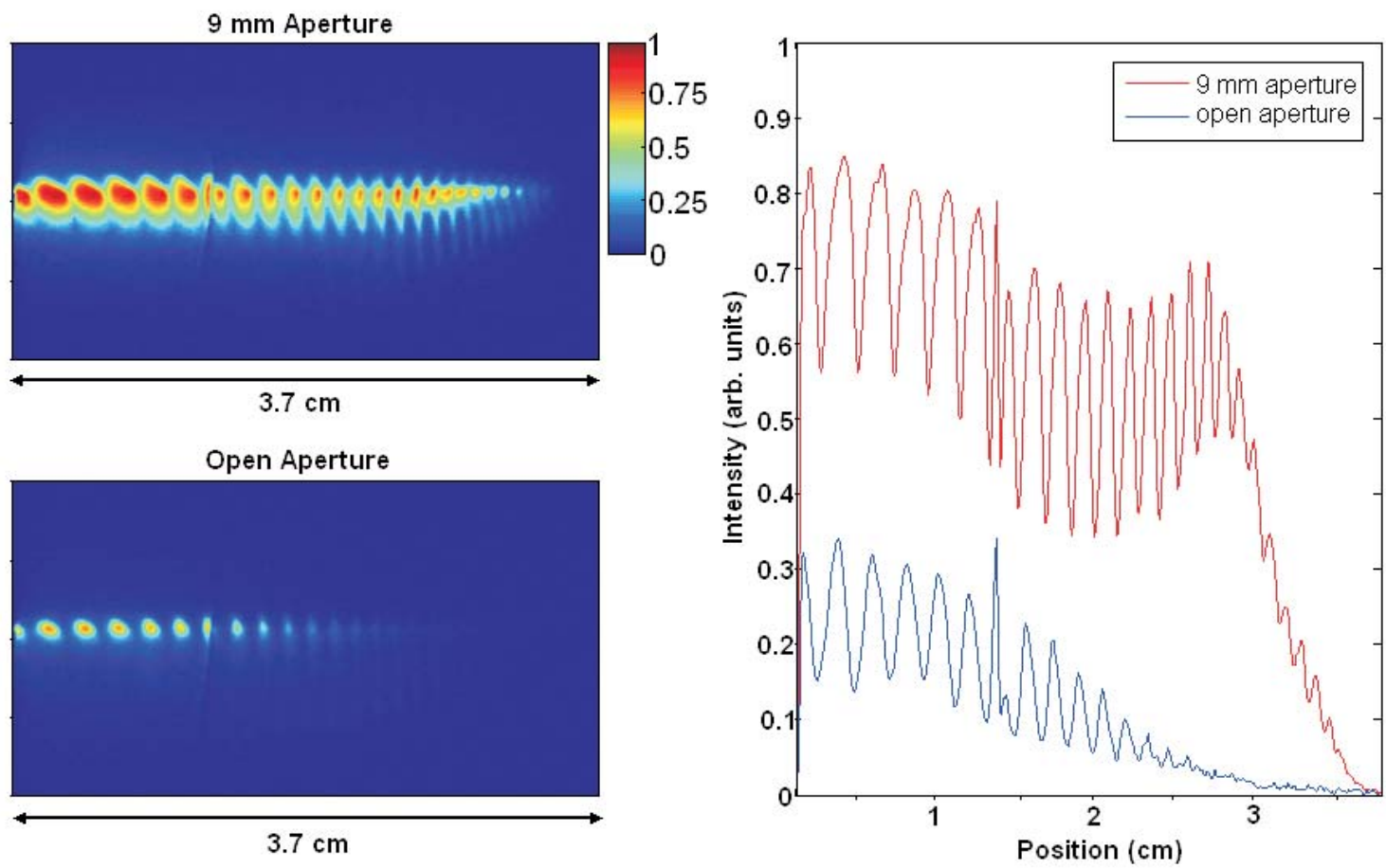

Figure 3.3 The intensity of harmonics with a slightly apertured beam was increased by almost a factor of 3 over the open aperture case. 
harmonics. When the beam is apertured to $9 \mathrm{~mm}$ the intensity at the focus decreases by a factor of 5 . This attenuates the peak intensity to $1.08 \times 10^{15} \mathrm{~W} / \mathrm{cm}^{2}$, which is just below the value at which helium readily ionizes, $1.5 \times 10^{15} \mathrm{~W} / \mathrm{cm}^{2}$. Images of the generated harmonics along with measured beam profiles are seen in Figs. 3.4, 3.5, 3.6 , and 3.7 .

It was possible to perform a scan over $9 \mathrm{~cm}$ by putting the original gas cell near one end of its range, after we scanned through the $5 \mathrm{~cm}$, the vacuum chamber was opened and a new gas cell was placed $4 \mathrm{~cm}$ earlier upstream. This gives a total range of $9 \mathrm{~cm}$, with a $1 \mathrm{~cm}$ overlap between measurements. When a new gas cell is used, the laser must self drill a new exit hole in the molybdenum foil. The centimeter overlap ensured consistency between the two cells.

The images reveal that best harmonics occur when the beam profile is nearly a 'top-hat' shape. For foil positions further away, either upstream or downstream, the harmonics gradually drop off, as does the 'top-hat' shape. Because the 'top-hat' shape is associated with filamentation, this suggests that harmonics are improved when a filament is present in the focus. When the harmonics are brightest the beam has a plateau shaped profile. This profile gradually becomes Gaussian as the harmonics decrease, regardless of whether upstream or downstream of the focus. The filament is centered at $0.5 \mathrm{~cm}$ (upstream from best focus in vacuum.) The filament is discussed more in Section 3.4.

\subsection{Laser Propagation in Gas Versus in Vacuum}

In this section images of the laser focus taken with the cell evacuated are compared to those taken in helium. Of course, no harmonics are generated in vacuum so they are not shown. In these figures the image on the left corresponds to the beam in 


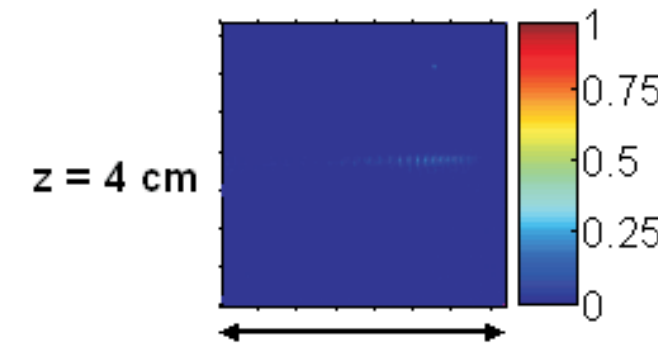

$3.7 \mathrm{~cm}$
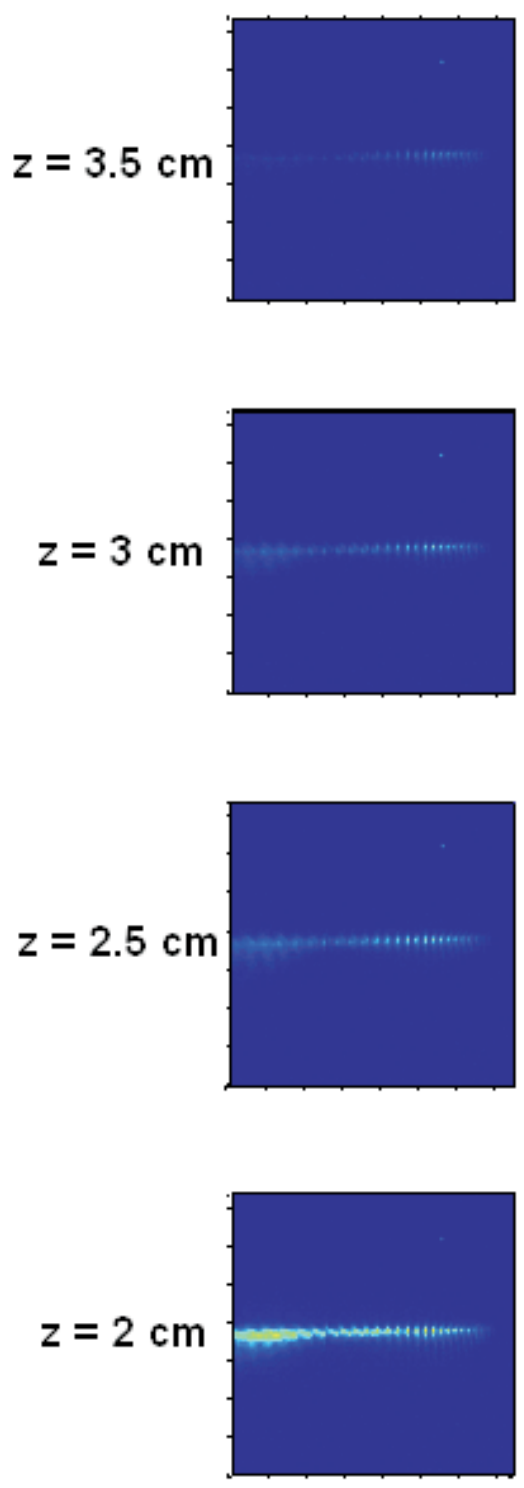
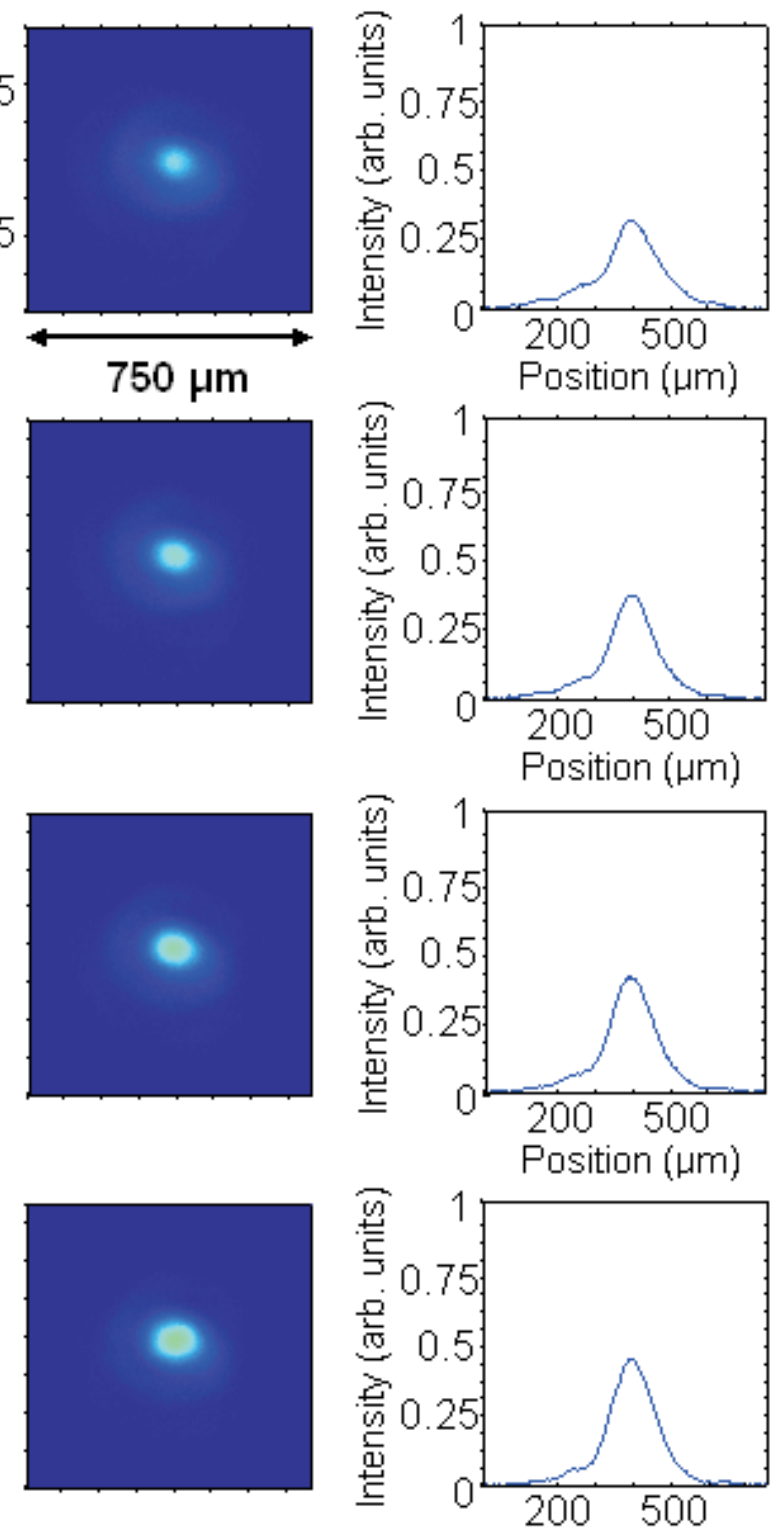

Position ( $\mu \mathrm{m})$
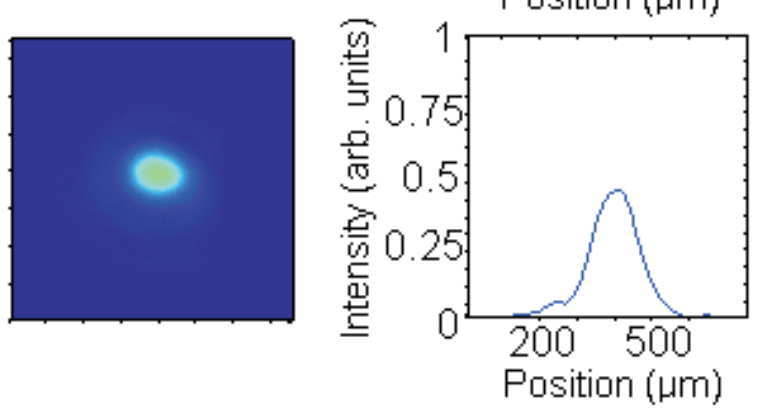

Figure 3.4 Harmonics generated at various foil positions along with beam profile images. 


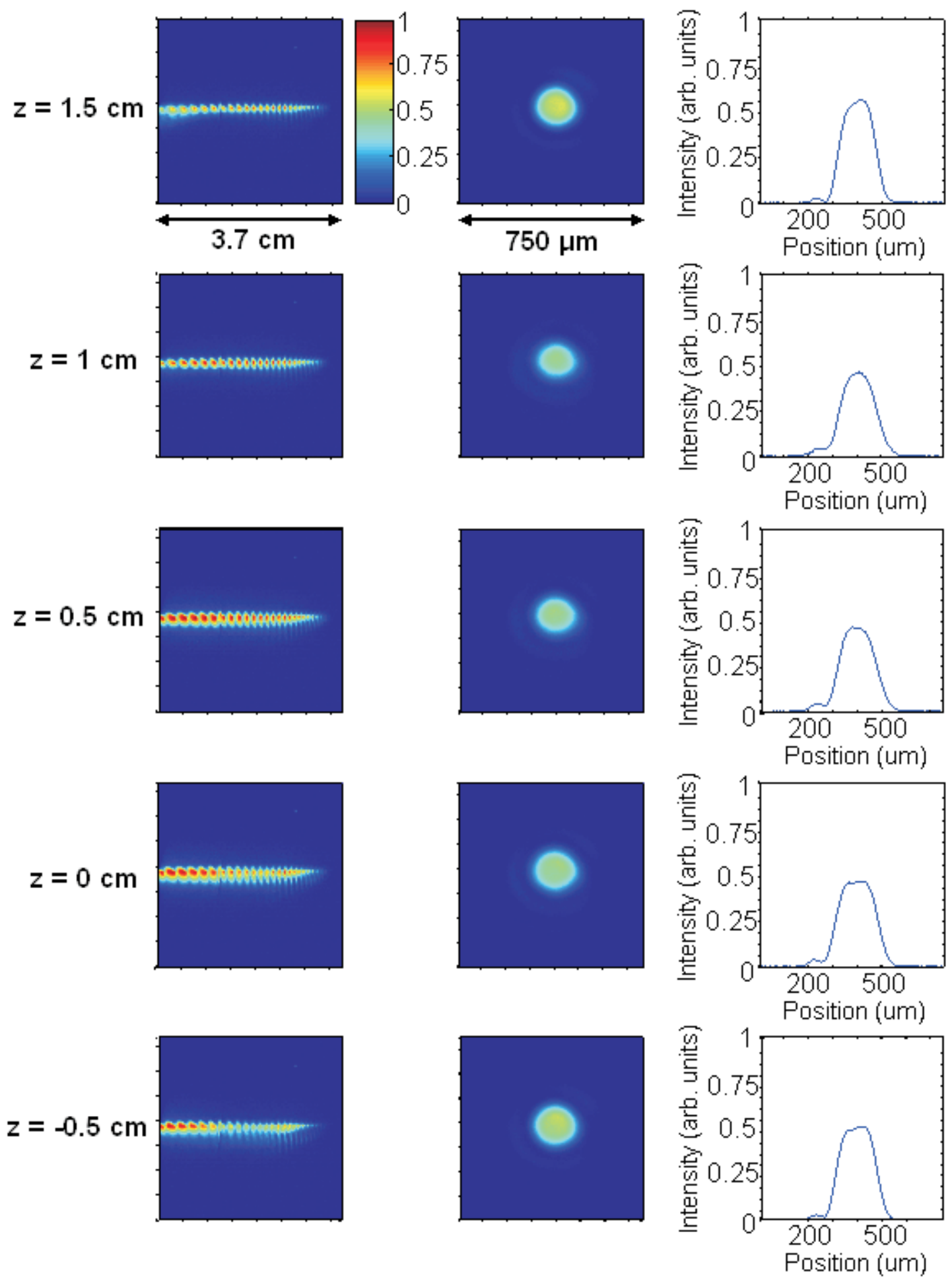

Figure 3.5 Harmonics generated at various foil positions along with beam profile images. 


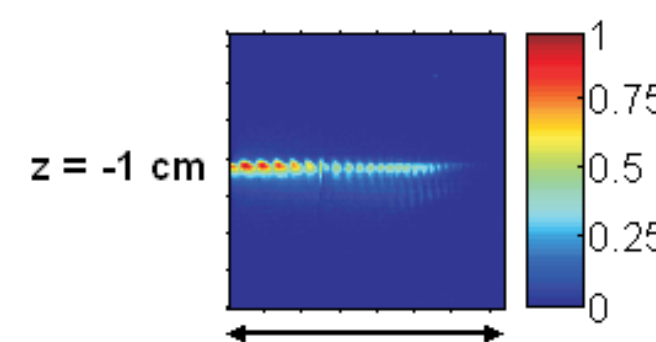

$3.7 \mathrm{~cm}$
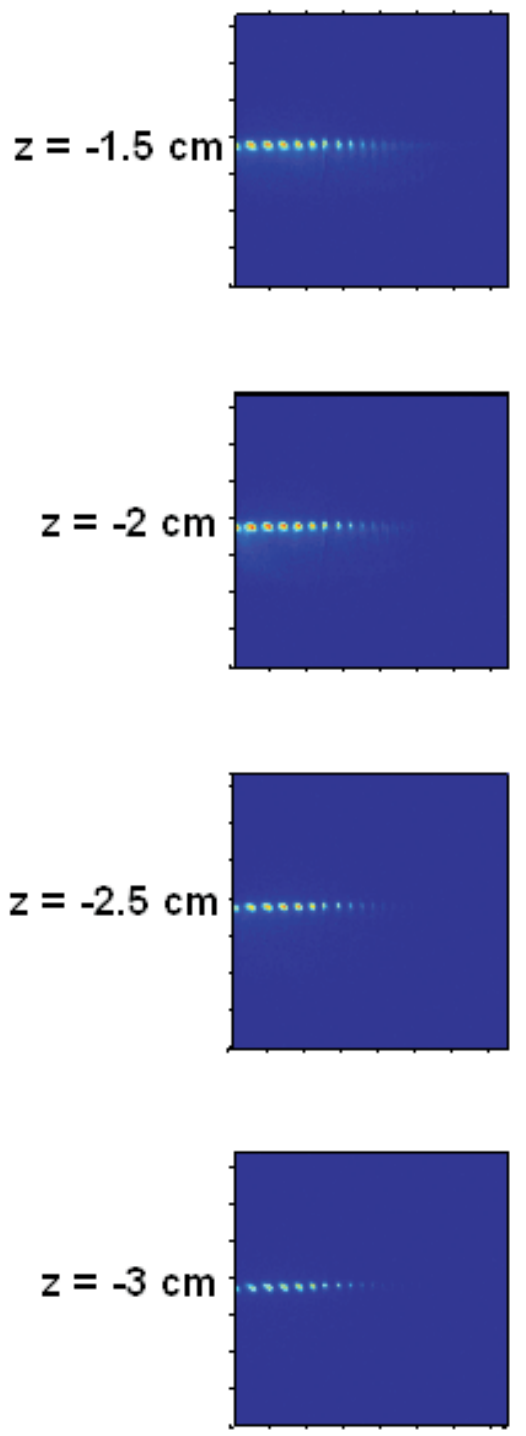

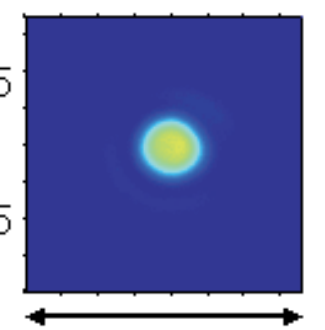

$750 \mu \mathrm{m}$
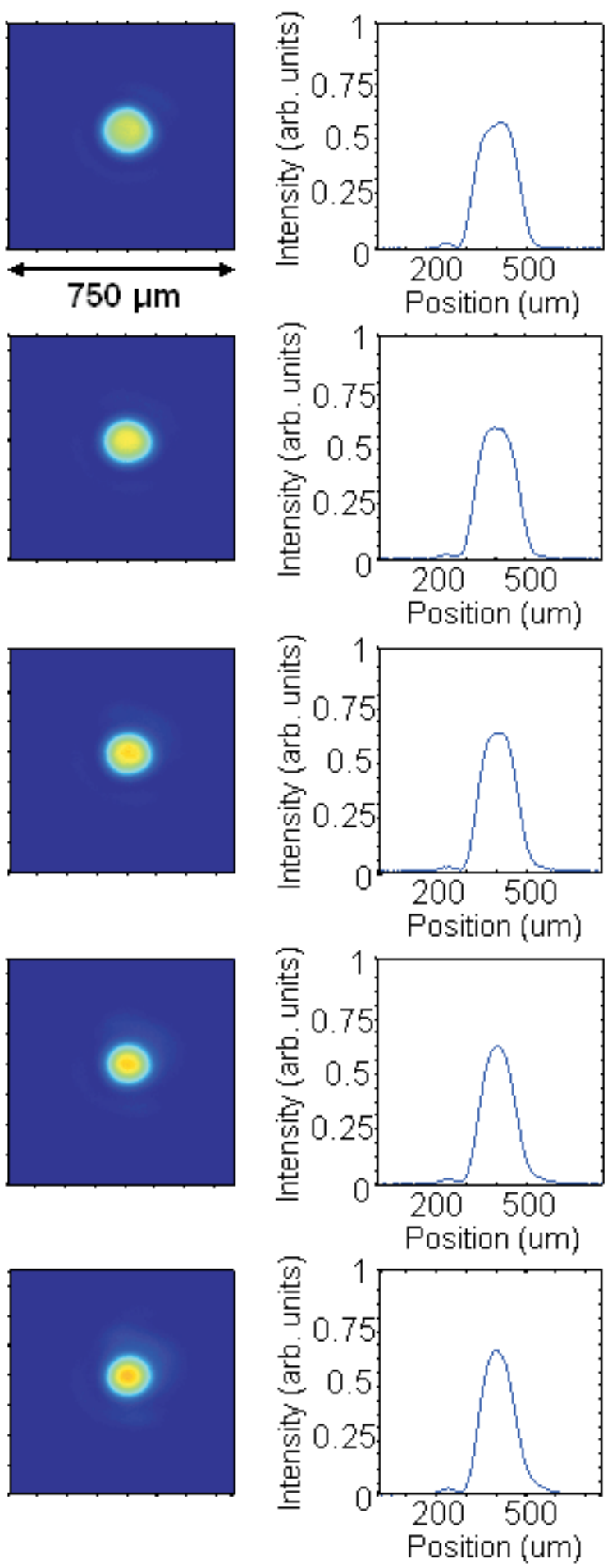

Figure 3.6 Harmonics generated at various foil positions along with beam profile images. 


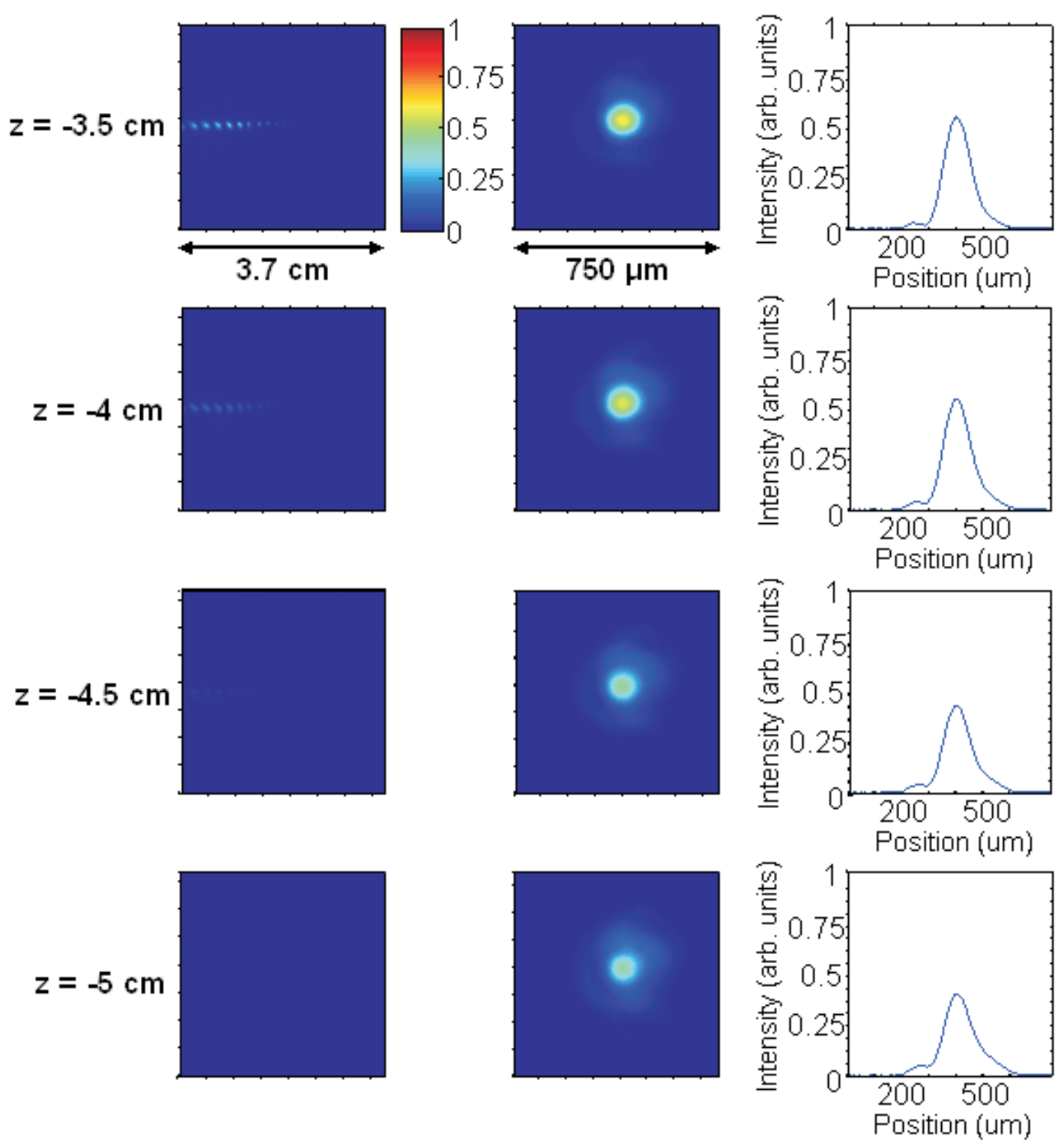

Figure 3.7 Harmonics generated at various foil positions along with beam profile images. 
helium with an accompanying line-out profile that shows the spatial shape of the beam and the image on the right corresponds to the beam focused in vacuum with its accompanying line-out. For vacuum scans, the gas-cell tube and foil were removed from the vacuum chamber since they were not needed. The camera positions for the vacuum scan were the same as they were in helium. In Figs. 3.8, 3.9, 3.10, and 3.11 negative numbers correspond to foil positions upstream of the laser focus, while positive numbers correspond to foil positions downstream of the laser focus, located $100 \mathrm{~cm}$ from the concave mirror.

The key difference between the two beams is the profile shape. In vacuum the shape of the beam profile is Gaussian and reaches its peak intensity at the focus. The beam focused in helium begins Gaussian and looks similar to the vacuum beam, but the two beams differ in a $5 \mathrm{~cm}$ range centered at the focus. The beam in helium becomes wider with a 'top-hat' profile. A comparison of the widths of the two beams is given in the next section. This 'top-hat' profile goes away as the imaged plane gets further than $\sim 2.5 \mathrm{~cm}$ from the focus. The presence of this 'top-hat' feature has been reported to be one of the evidences of filamentation [18], [19], and [20].

The 'top-hat' profile is explained by the same two effects that cause filamentation. In the outer radius of the beam there are no free electrons, which would disperse the beam, therefore the laser beam tries to focus due to the nonlinear self focusing in the gas. However, the generation of free electrons in the inner radius tends to disperse the beam, acting like a negative lens.

\subsection{Beam-Width Measurements}

The full-width-at-half-maximum (FWHM) diameters of the laser beam in helium and vacuum are compared in Fig. 3.12. The aperture for both beams is set at $9 \mathrm{~mm}$. The 


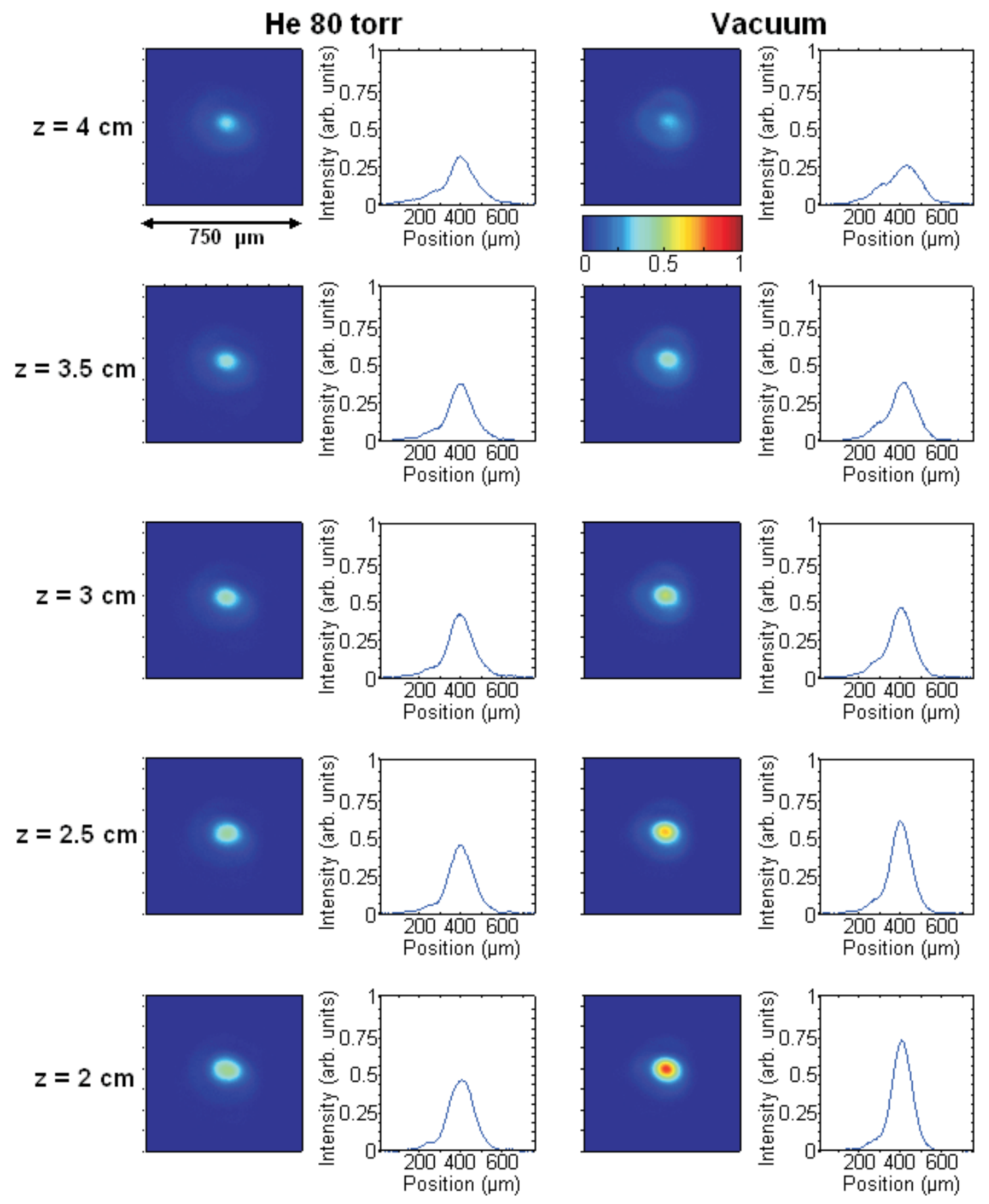

Figure 3.8 Laser beam profile for helium and vacuum at various foil positions relative to the beam focus. 

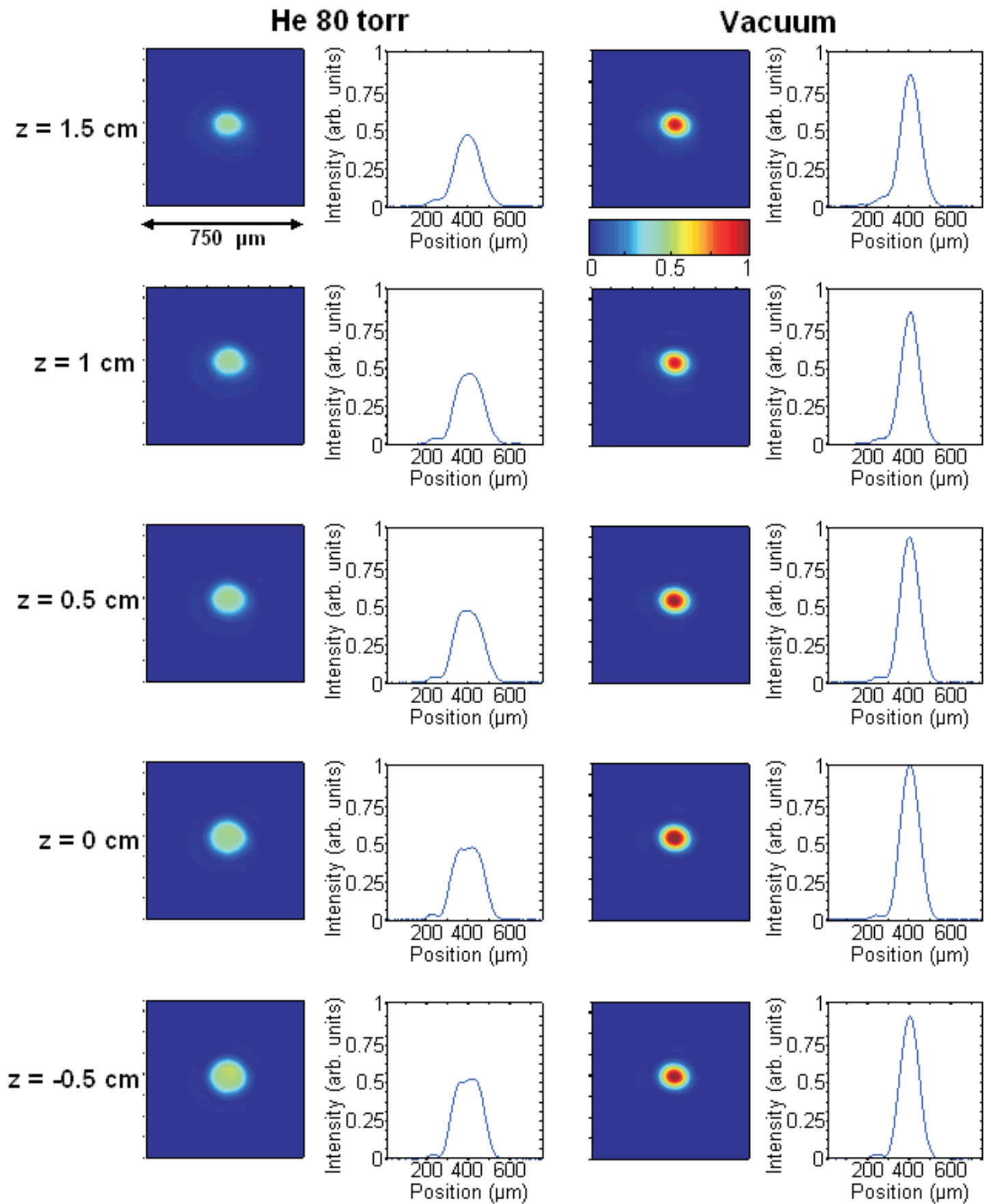

Figure 3.9 Laser beam profile for helium and vacuum at various foil positions relative to the beam focus. 


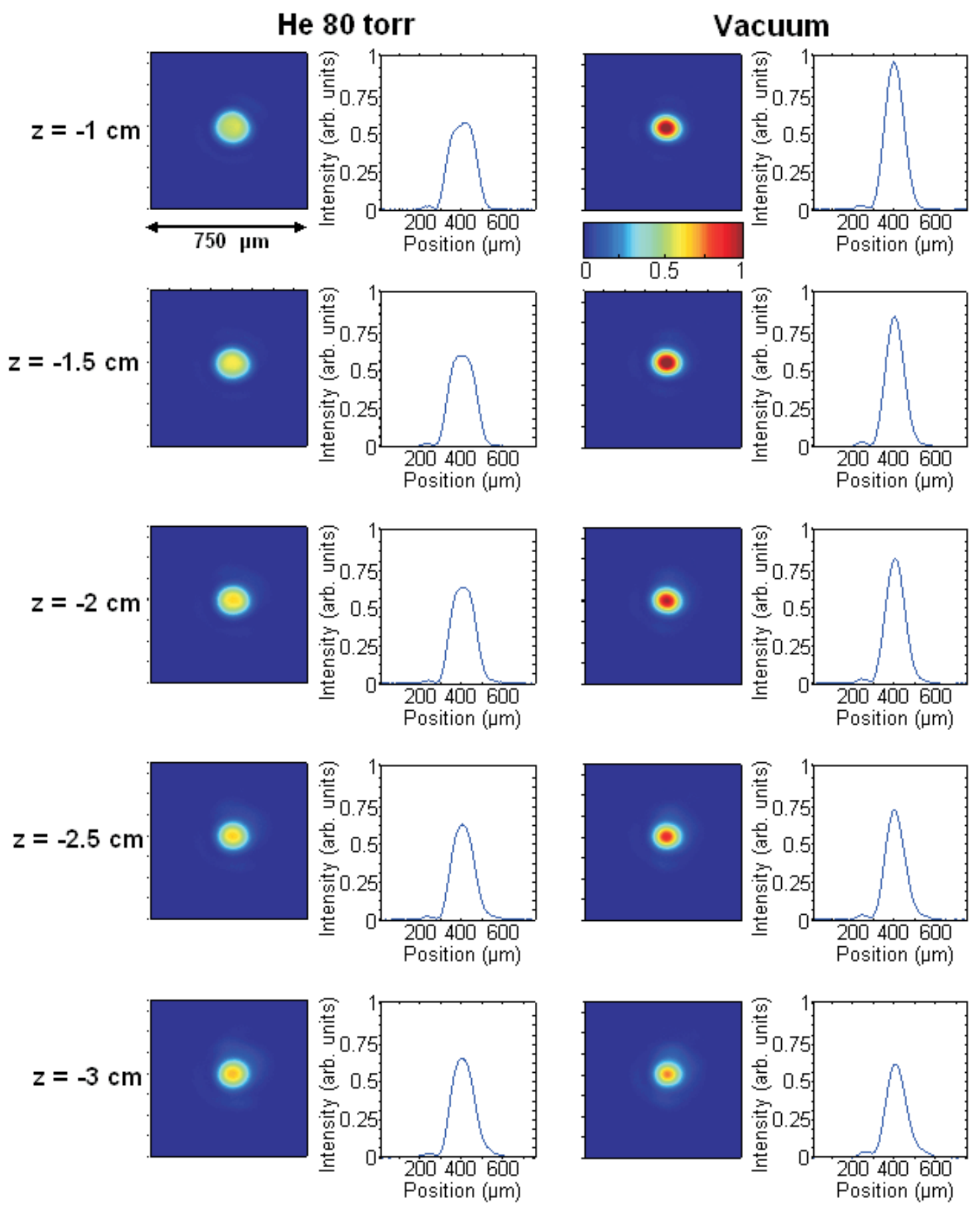

Figure 3.10 Laser beam profile for helium and vacuum at various foil positions relative to the beam focus. 


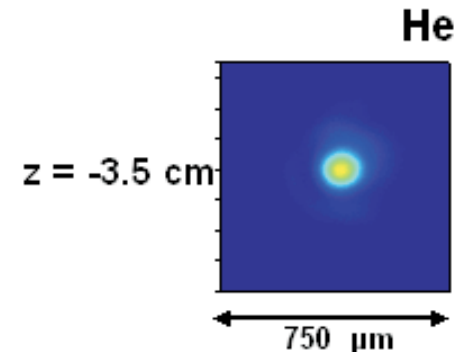

He 80 torr
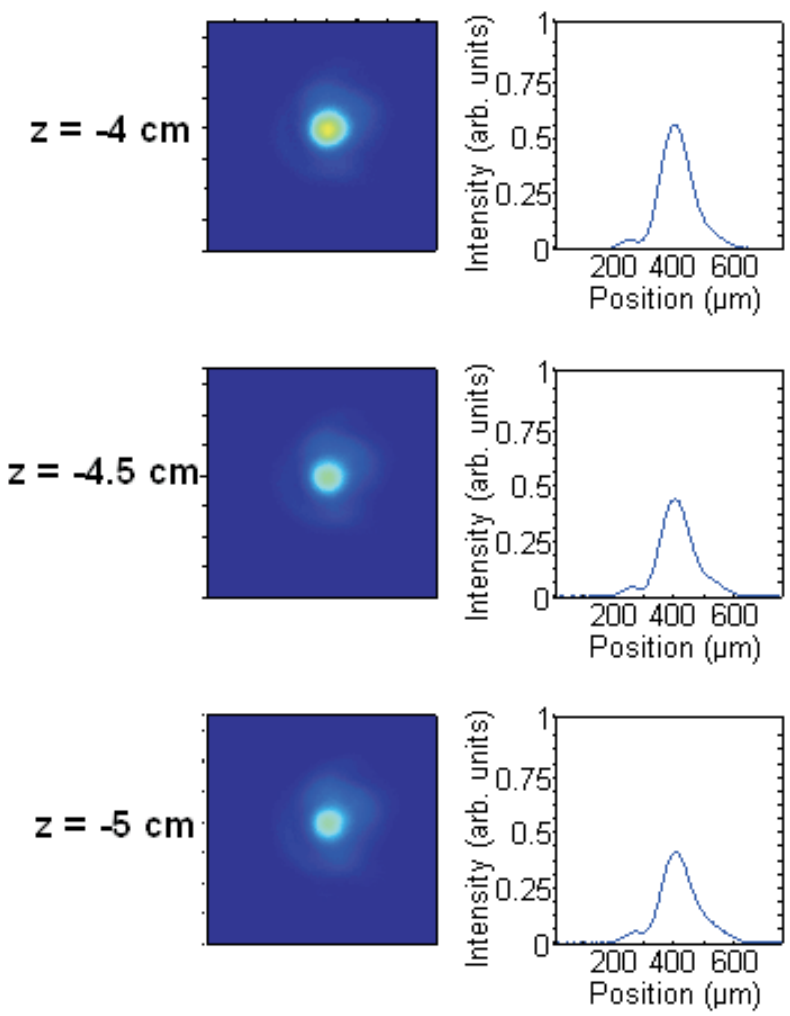

\section{Vacuum}
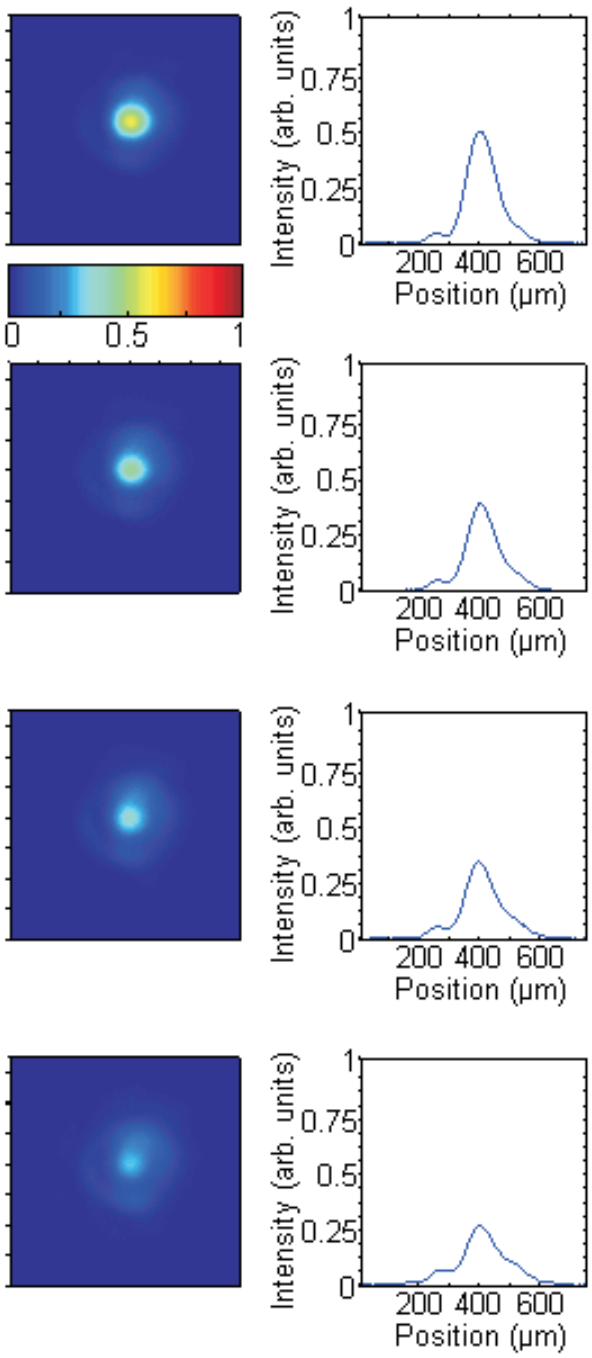

Figure 3.11 Laser beam profile for helium and vacuum at various foil positions relative to the beam focus. 


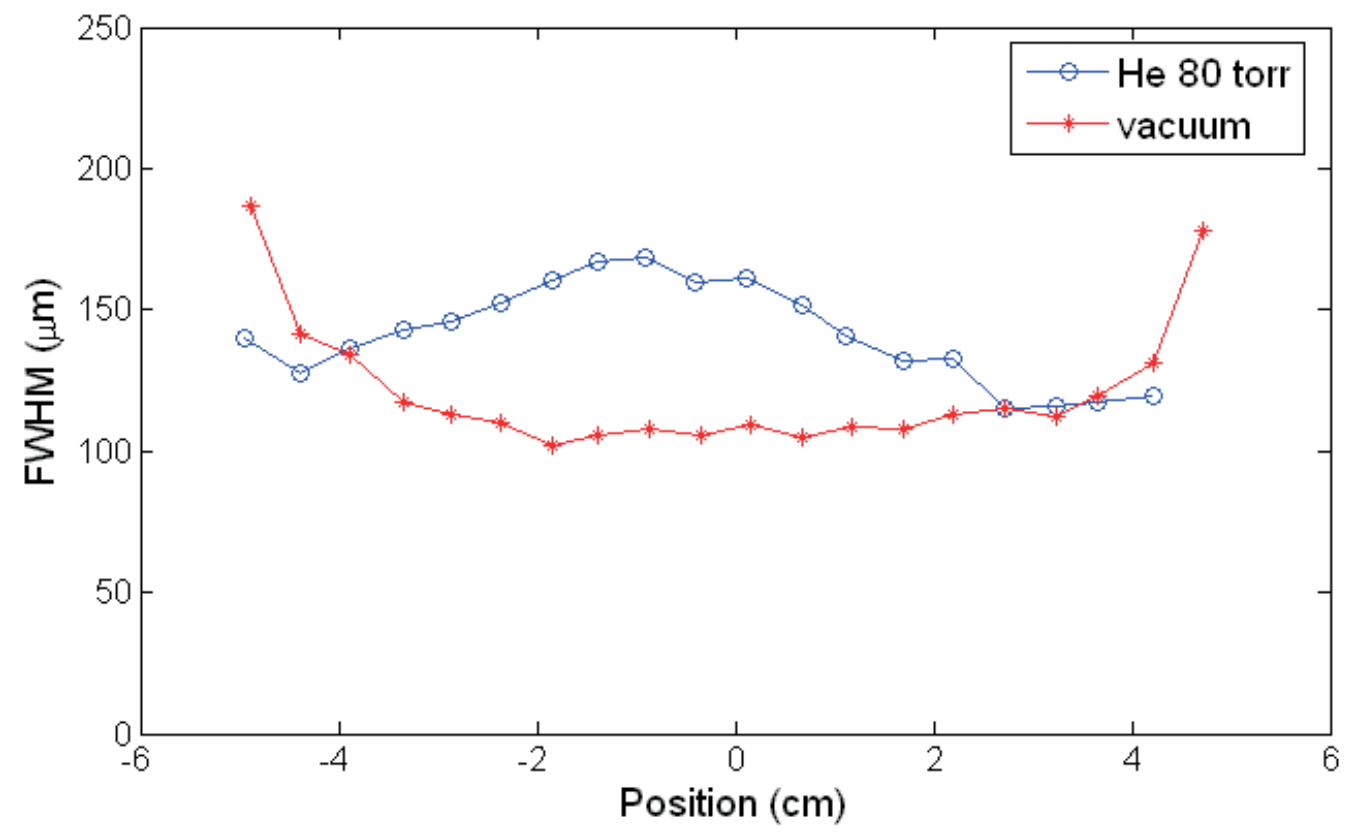

Figure 3.12 A graph of FWHM for both helium and vacuum for various foil positions.

FWHM value for the beam focused in vacuum reaches a smaller value than it does for helium. This is due to the interaction with helium. The dispersion due to ionized electrons limits how tightly the beam can focus in helium.

In Fig. 3.12 a double focus is observed. The laser beam initially begins to focus, but the intensity becomes high enough that it defocuses due to ionization. It is through the region where the beam has grown and held relatively constant (FWHM $\sim 150 \mu \mathrm{m})$ for several centimeters that we achieve best harmonics. Eventually intensity-dependent losses deplete the energy in the channel and the process becomes unbalanced. The length of the plasma generated in the focus is approximately 4-6 cm. If the distance between the two lowest FWHM points for helium in Fig. 3.12 are indicative of the length of the filament then it would extend over a region of $7 \mathrm{~cm}$.

Kosareva et al. [22] report having seen multiple foci in their ultra short laser 
pulses in air. These foci extended the focus of their beam to several meters in air while maintaining a beam radius less than $100 \mu \mathrm{m}$. Although the scaling is different for our situation, the formation of multiple foci resembles what we see in our helium data in Fig. 3.12. 


\section{Chapter 4}

\section{Auxiliary Measurements}

We characterized the laser pulse duration, the spectral content of the laser before and after interaction with the gas, as well as the energy converted into high-harmonic beams.

\subsection{Pulse Duration}

In addition to looking at the imaged focus when the aperture was optimized at $9 \mathrm{~mm}$, we compared the temporal duration of the pulse optimized for best harmonic production to the shortest attainable temporal duration. Fig. 4.1 shows a comparison between the two auto-correlation traces. The temporal duration of the pulse that was best for harmonics is wider by about $16 / \sqrt{2}$ fs. However, the pulse that we use for auto-correlation travels through a $1 / 4$ in thick piece of fused silica on its way to the nonlinear crystal. Going through the fused silica introduces a chirp on the pulse that is about right to account for the pulse duration seen. Thus, it appears that the pulse is likely optimally compressed as it approaches the laser focus. On the other

hand, it is possible that it is negatively chirped by double the amount of positive 


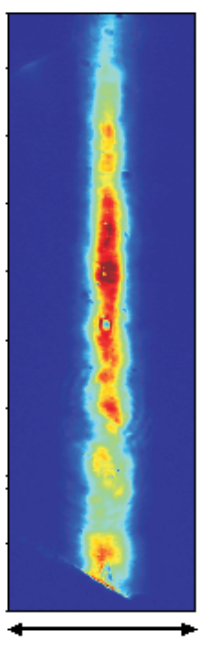

$1.5 \mathrm{~mm}$
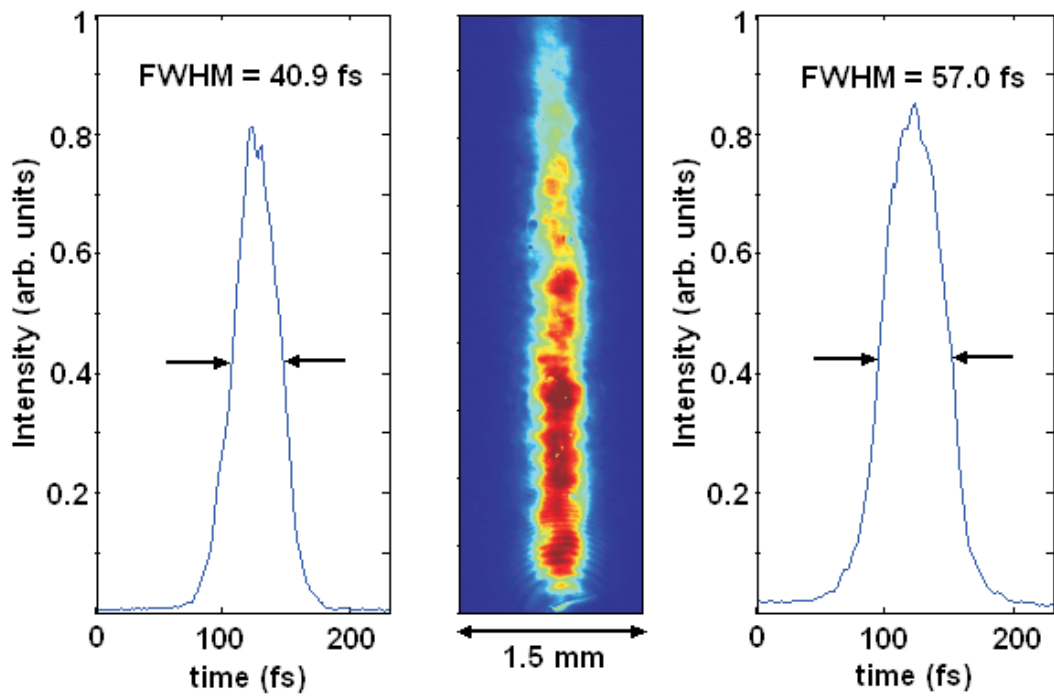

Figure 4.1 Comparison of the temporal pulse duration optimized in air(left) versus best harmonic compression(right).

chirp introduced by the fused silica before the auto-correlation measurement. Since the amount of chirp in dispute is small, it is fair to say that the pulse used in optimal harmonic production is close to a well-compressed pulse.

\subsection{Laser Focus Spectral Content}

The spectral characteristics of the laser focused in vacuum and in helium were measured. An Ocean Optics spectrometer (model number USB2000) with a 50- $\mu$ m-core fiber optic was used to capture spectral information at different locations in the laser focus. Neutral-density filters were used to attenuate the laser beam for optimal instru-

ment exposure. The fiber was attached to two linear translation stages to accurately control its position in the imaged focus. One stage allows for horizontal movement and the other for vertical movement.

We observed spatial chirp in our laser beam through the use of the spectrometer. As the fiber was horizontally swept through the focus, one side of the spectrum was 

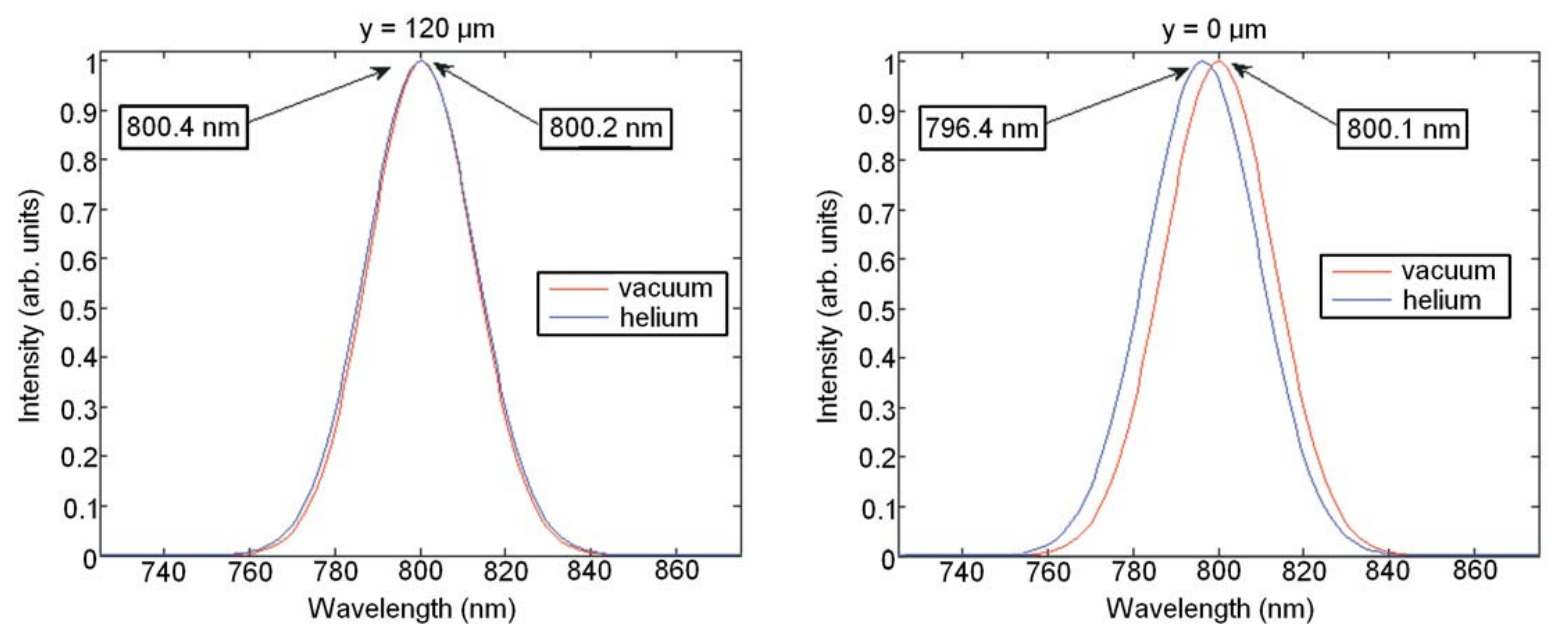

Figure 4.2 Spectral content of imaged laser focus in both helium and vacuum at outer radius of beam and in center of beam.

blue shifted while the opposite side was red-shifted. We were able to minimize the spatial chirp by appropriately adjusting the compression gratings. Because slight spatial chirp is observed as the fiber is swept horizontally through the focus, for capturing the radial spectral content of the laser focus, the fiber was swept vertically through the focus.

Two things that we look for in the focus of the laser beam is radial variations in the spectrum due to ionization as well as an overall shift of the spectrum compared to vacuum. To have a reference of our laser spectrum in the absence of any unexplained nonlinear effects, we first swept a spectrometer vertically through the imaged laser focus. A similar spectral scan was performed of the imaged beam under optimal harmonic generation conditions (i.e. aperture diameter, gas pressure and exit foil location). Fig. 4.2 shows the results from the spectral scans. The data has been normalized so that the helium blue-shift can easily be seen. The fiber optic position, $y=120 \mu \mathrm{m}$, refers to the radial position in the actual focus rather than the imaged one.

The laser beam is centered around $800 \mathrm{~nm}$ with a FWHM of $35 \mathrm{~nm}$. The vacuum 
spectrum exhibits no shift in its spectrum as the fiber is scanned vertically through the focus. However, the helium spectrum exhibits a shift of around $4 \mathrm{~nm}$ for the center of the focus compared to vacuum. The profile of the beam at this position is 'top hat', it is the flattened portion of the beam that exhibits the blue-shift, see Fig. 3.5. The blue-shifting is due to the time-dependent index of refraction with the creation of the plasma, which occurs most in the laser center.

\subsection{Harmonic Energy Measurement}

Conversion efficiency is the ratio of the output energy of the individual harmonics to the input energy of the generating laser. We used an AXUV-100 photodiode from International Radiation Detectors Inc.(IRD) to determine the energy of the generated high-order harmonics (orders 41 - 91). For more information see the senior thesis of Mark Adams [28]. This is a similar setup to that used by Takahashi et al. [12].

Because the efficiency of our EUV diffraction grating is not known, we eliminate the grating and measure the total energy of all harmonics present in the beam (that cause a response on the detector). Because the harmonics are embedded in the residual laser beam, we use a $0.2 \mu \mathrm{m}$-thick $\mathrm{Zr}$ filter to block the laser light. It also blocks the low-order harmonics. The photodiode was positioned inside a cylindrical housing made of aluminum, directly behind the filter and axially aligned with it, see Fig. 4.3. A coaxial cable, which reduces electrical noise, is used to connect the photodiode to the oscilloscope.

For our energy measurement, the diode is placed in the path of the harmonics before the grating. The high-harmonic photons that make it through the filter excite electrons to the conduction band of the diode, which results in a voltage governed by the intrinsic capacitance of the photodiode and the cabling to the oscilloscope. As 


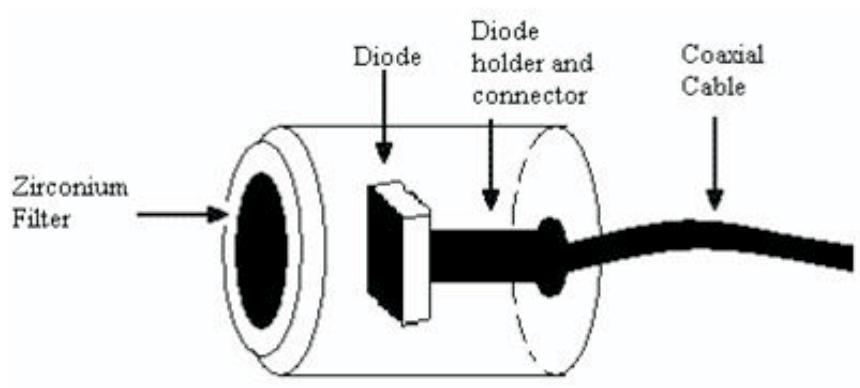

Figure 4.3 Filter and photodiode assembly. Harmonic light enters the cylinder through a zirconium filter. The aluminum cylindrical housing prevents stray light from reaching the diode [28].

seen in Fig. 4.4, we saw approximately $29 \mathrm{mV}$ peak voltage for our measurement. The charge in the device immediately begins to discharge through the $50 \Omega$ termination of the oscilloscope. The capacitance is found via $\tau=R C$, where $R$ represents the internal resistance of the oscilloscope, which was set to $50 \Omega$. The time constant $\tau$ is defined as the time for the diode to discharge to $1 / e$ of the peak voltage. From Fig. 4.4 , we see this voltage decay is about $0.6 \mu$ s. We calculate the capacitance to be $1.2 \times 10^{-8} \mathrm{~F}$.

We also took a measurement of the photodiode with the gas cell evacuated but with the laser light hitting the filter directly. Fig. 4.5 shows the case, which indicates that the electrical noise was about $1 \mathrm{mV}$, which we ignore in our calculations.

The filter/photodiode combination is sensitive only to harmonics in the range from $41^{\text {st }}$ to $91^{\text {st }}$ harmonic, the highest produced. We make the simplifying assumption that the energy $E_{t}$ held in each harmonic is approximately the same. This is reasonable, given the uniform brightness of the harmonics seen in Fig. 2.5. The zirconium filter is 


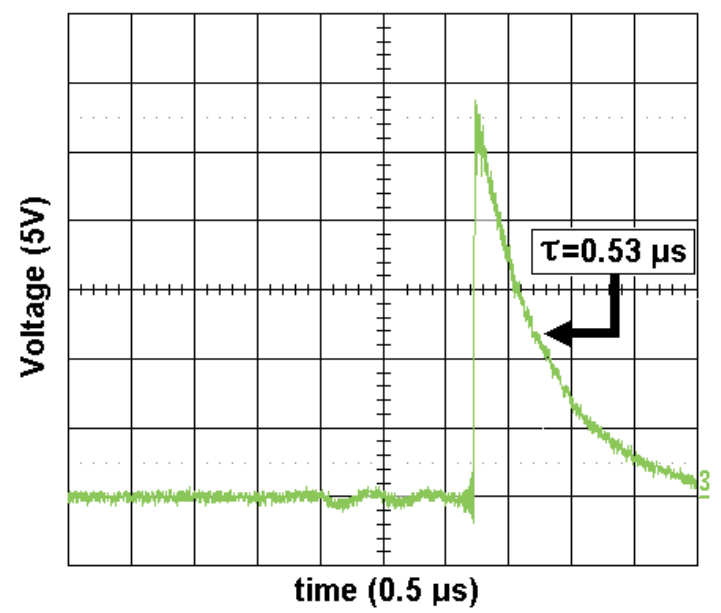

Figure 4.4 Voltage induced in the photodiode.

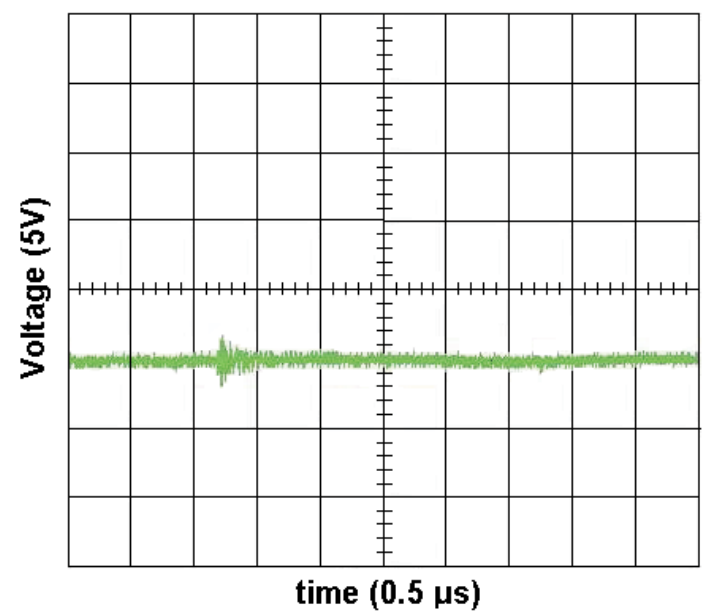

Figure 4.5 Voltage induced in the photodiode due to electrical noise. 


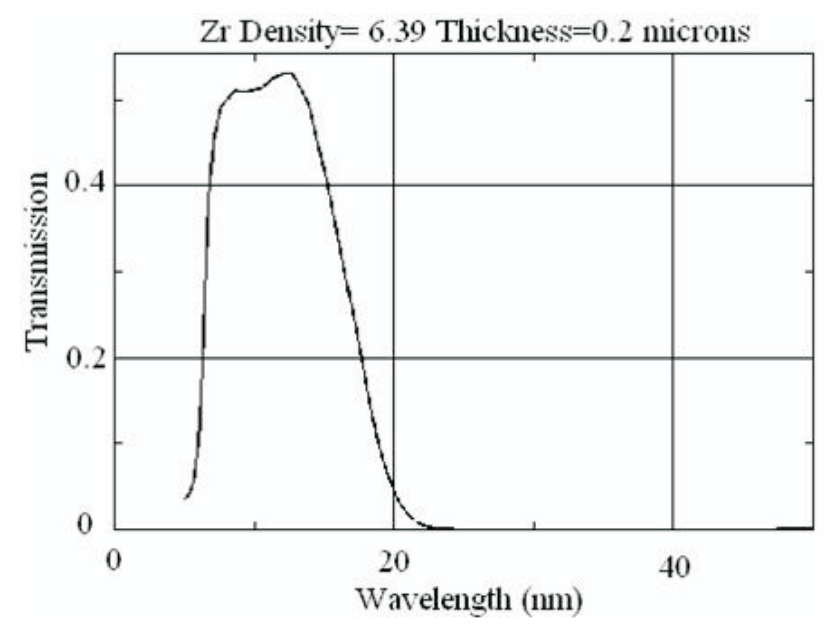

Figure 4.6 Zirconium filter transmission curve for short wavelength light $[29]$.

wavelength-dependent, however, which must be taken into account when calculating transmitted energies. The spectral response of the filter is seen in Fig. 4.6.

The energy of each harmonic arriving to the detector $E_{n}^{\prime}$ is affected by the filter transmission according to $E_{n}^{\prime}=T_{n} E_{n}$, where $E_{n}$ is the energy in the $n^{\text {th }}$ harmonic and $T_{n}$ is the transmission through the filter. The number of photons in each harmonic pulse arriving to the photodiode is determined by dividing the energy of each harmonic by the energy of a single photon:

$$
N_{n}=\lambda_{L} E_{n} / n h c
$$

Here $h$ is Planck's constant, $c$ is the speed of light, and $\lambda_{L}$ is the laser wavelength.

The average number of electrons that are promoted to the conduction band per photon depends on the harmonic order through the quantum efficiency $q_{n}$. The quantum efficiency is taken from a graph provided by the manufacturer of the photodiode, (see Fig. 4.7).

The total charge resulting from all harmonic orders is found from summing the quantum efficiency of the photodiode for each harmonic order times the number of 


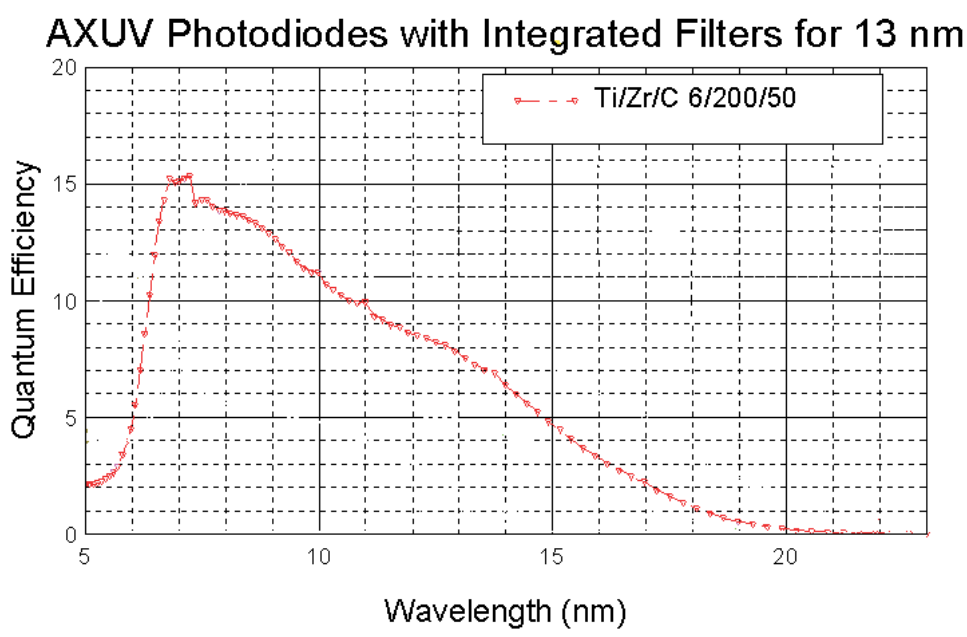

Figure 4.7 Quantum efficiency of photodiode provided by IRD Inc. [?].

photons for that particular order (as well as the charge of an electron):

$$
Q=e \sum q_{n} N_{n}
$$

Combining this equation with the number of photons arriving to the photodiode and the energy of each harmonic yields

$$
Q=\frac{e \lambda_{L} E_{n}}{h c} \sum \frac{q_{n} T_{n}}{n}
$$

We bring the harmonic energy $E_{n}$ outside the sum in accordance with our assumption that it is the same for all relevant orders. The sum is over odd integers from 41 to 91.

The charge is related to the capacitance of the diode through $Q=C V$, where $V$ is the peak voltage seen on the oscilloscope, in our case $29 \mathrm{mV}$. The charge on the capacitor is calculated to be $3.48 \times 10^{-10} \mathrm{C}$. From this result and from applying transmission values and quantum efficiencies, we calculate an energy $E_{n}$ of individual harmonics to be approximately $1 \mathrm{~nJ}$ for orders between 41 and 91 . This gives a total energy of about $26 \mathrm{~nJ}$ in the 26 orders present (odd). The conversion efficiency of the $4 \mathrm{~mJ}$ laser energy into individual harmonics is about $2 \times 10^{-7}$. 
The conversion efficiencies reported earlier in section 1.2.1 were for lower-order harmonics. We are achieving comparable efficiencies for higher ordered harmonics. This gives us confidence in the effectiveness of our gas cell configuration as a competitive method of HHG. 


\section{Chapter 5}

\section{Conclusions}

In summary, we have constructed an imaging apparatus that allows us to explore the laser beam spatial profile as it evolves at full power while interacting with gas during production of high-order harmonics. We adjusted parameters such as gas pressure and aperture diameter to optimize harmonic production. We measured the conversion

efficiency for our semi-infinite-gas-cell geometry to be approximately $2 \times 10^{-7}$, which is similar to those reported by other groups, although we have focused our attention on higher orders than those for which conversion-efficiency has been measured.

Images of the laser focus revealed the presence of filamentation when the conditions for optimal harmonic generation where present, namely the gas pressure at 80 torr and the beam aperture closed to $9 \mathrm{~mm}$. The images of the laser beam at 80 torr helium show the formation of a double focus, neither of which is as tightly focused as the beam in vacuum. This is direct evidence of filamentation. Kim et al. [20] present an image of their laser beam at one position when harmonic generation is optimized. However, we perform an extensive scan of the laser beam showing the clear formation of a filament. Best harmonics are attained when the exit foil of the gas cell is positioned near the middle of the filament where the beam diameter is 
greater. We also observe the 'top-hat' beam profile in this same region over a distance of several centimeters. This is in agreement with what Tosa et al. predicted [18] and Kim et al. reported [20].

A spectral measurement in the imaged laser focus revealed a minor blue-shift of the laser spectrum when 80 torr of helium was present in the gas cell, compared to vacuum. A blue-shift was observed in the focus of the harmonic generating beam in helium. The vacuum beam remained mostly centered around $800 \mathrm{~nm}$ through the entire focus. The helium beam was slightly blue-shifted by about $5 \mathrm{~nm}$. A slight blue-shift is expected in the focus due to the time-dependent index of refraction of the generated plasma, which changes the frequency while keeping the wavelength the same.

Record conversion efficiencies of $10^{-4}$ have been reported by Takahashi et al. [12] for harmonics generated in xenon. These were much lower orders than what we have characterized. For our high ordered harmonics (41-91), we calculate a conversion efficiency of $2 \times 10^{-7}$ for individual harmonics being generated by $4 \mathrm{~mJ}$ pulses. Higher ordered harmonics typically have lower conversion efficiencies which currently limits their applications.

As was mentioned in section 1.1, Professors Allred and Turley design and fabricate multilayer coatings for EUV optics. Because our high-order harmonics are polarized and have sufficiently high energy, they will be used as a EUV source for determining optical constants of Allred's and Turley's EUV optics. Given the dynamic range of our setup, the high-order harmonics are capable of measuring reflectances as low as 0.5\% [30]. Niki Brimhall, a current graduate student who has contributed significantly to the work presented here, will construct and test a polarimeter based on the harmonics generated in the regime outlined in this thesis. This polarimeter will be a valuable asset to BYU by providing a local and less-expensive alternative to visiting 
a synchrotron source. 


\section{Bibliography}

[1] K. David, "Intel's EUV lithography process line," http://www.intel.com/ technology/silicon/lithography.html (2004).

[2] "ALS Quick Facts," http://www-als.lbl.gov/als/aboutals/alsquickfacts.html (2006).

[3] J. Jonkers, "High power extreme utlra-violet light sources for future lithgraphy," XXVIIth ICPIG, Eindhoven, the Netherlands pp. 16-20 (2005).

[4] B. Hansson, O. Hemberg, and H. Hertz, "Characterization of a liquid-xenonjet laser-plasma extreme-ultraviolet source," Rev. Sci. Instrum. 75, 2122-2129 (2004).

[5] http://euv.lpl.arizona.edu/euv/ (2006).

[6] A. McPherson, G. Gibson, H. Jara, U. Johann, T. S. Luk, I. A. McIntyre, K. Boyer, and C. K. Rhodes, "Studies of multiphoton production of vacuumutraviolet radiation in the rare gases," J. Opt. Soc. Am B 4, 595-601 (1987).

[7] A. L'Hullier and P. Balcou, "High-order harmonic generation in rare gases with a 1-ps 1053-nm laser," Phys. Rev. Lett. 70, 774-777 (1993). 
[8] A. Rundquist, C. D. III, Z. Chang, C. Herne, S. Backus, and M. M. adn Henry Kapteyn, "Phas-Matched generation of coherent soft x-rays," Science 280, 1412-1415 (1998).

[9] Y. Tamaki, J. Itatani, Y. Nagata, M. Obara, and K. Midorikawa, "Highly efficient phase-matched high-order harmonic generation in self guided laser beam," Phys. Rev. Lett. 82, 1422-1425 (1999).

[10] E. Takahashi, Y. Nabekawa, T. Otsuka, M. Obara, and K. Midorikawa, "Generation of highly coherent submicrojoule soft x-rays by high-order harmonics," Phys. Rev. A 66, 0021802(R) (2002).

[11] E. Takahashi, Y. Nabekawa, and K. Midorikawa, "Low divergence coherent soft x-ray source at $13 \mathrm{~nm}$ by high-order harmonics," Appl. Phys. Lett. 84, 4-6 (2004).

[12] E. Takahashi, Y. Nabekawa, and K. Midorikawa, "Generation of 10- $\mu$ J coherent extremem-ultraviolet light by use of high-order harmonics," Opt. Lett. 27 (2002).

[13] Lasers, 1st ed. (John Wiley and Sons, NY, 1988).

[14] J.F.Hergott, M. Kovacev, H. Merdji, C. Hubert, Y. Mairesse, E. Jean, and P. Breger, "Extreme ultraviolet high-order harmonic pulses in the microjoule range," Phys. Rev. A 66, 021801(R) (2002).

[15] H. Milchberg, C. D. III, and T. McIlrath, "High-order frequency conversion in the plasma wavequide," Phys. Rev. Lett. 75, 2494-2497 (1995).

[16] C. D. III, A. Rundquist, S. Backus, C. Herne, M. Murnane, and H. Kapteyn, "Phase matching of high-order harmonics in hollow waveguides," Phys. Rev. Lett. 83, 2187-2190 (1999). 
[17] Y. Tamaki, Y. Nagata, M. Obara, and K. Midorikawa, "Phase-matched highorder harmonic generation in a gas-filled hollow fiber," Phys. Rev. A 59, 40414044 (1999).

[18] V. Tosa, E. Takahashi, Y. Nabekawa, and K. Midorikawa, "Generation of highorder harmonics in a self-guided beam," Phys. Rev. A 67, 063817 (2003).

[19] E. Takahashi, V. Tosa, Y. Nabekawa, and K. Midorikawa, "Experimental and theoretical analyses of a correlation between pump-pulse propagation and harmonic yield in a long-interaction medium," Phys. Rev. A 68, 023808 (2003).

[20] H. Kim, I. Kim, V. Tosa, Y. Lee, and C. Nam, "High brightness harmonic generation at 13 nm using self-guided and chirped femtosecond laser pulses," Appl. Phys. B 78, 863-867 (2004).

[21] "Effects of a gas medium on ultrafast laser beam delivery and materials processing," J. Opt. Soc. Am. B .

[22] O. Kosareva, V. Kandidov, A. Brodeur, C. Chien, and S. Chin, "Conical emission from laser-plasma interactions in the filamentation of powerful ultrashort laser pulses in air," Opt. Lett. 22, 1332-1334 (1997).

[23] M. Mlejnek, E. Wright, and J. Moloney, "Dynamic spatial replenishment of femtosecond pulses propagating in air," Opt. Lett. 23, 382 (1998).

[24] J. Philip, C. Amico, G. Cheriaux, A. Couairon, B. Prade, and A. Mysyrowicz, "Amplification of femtosecond laser filaments in Ti:sapphire," Phys. Rev. Lett. 95, $163901(2005)$.

[25] A. Braun, G. Korn, X. Liu, D. Du, J. Squier, and G. Mourou, "Self-channeling of high-peak-power femtosecond laser pulses in air," Opt. Lett. 20, 73-75 (1995). 
[26] J. Sutherland, "Phase-matching optimization of laser high-order harmonics generated in a gas cell," MS Thesis, Brigham Young University p. Brigham Young University (2005).

[27] E. Christensen, "Direct measurements of absorption rates for laser high-order harmonics generated in helium and neon," Senior Thesis p. Brigham Young University (2005).

[28] M. Adams, "Measuring absolute brightness of laser generated high harmonic emission," Senior Thesis p. Brigham Young University (2005).

[29] "University of California - Berkeley," http://www-cxro.lbl.gov/opticalconstants/ filter2.html (2006).

[30] S. Voronov, "Controlling laser high-order harmonic generation using weak counter-propagating light," PhD Dissertation p. Brigham Young University (2003). 